\title{
Search for the Higgs boson in lepton, tau, and jets final states
}

V. M. Abazov, ${ }^{32}$ B. Abbott, ${ }^{68}$ B. S. Acharya, ${ }^{26}$ M. Adams,${ }^{46}$ T. Adams, ${ }^{44}$ G. D. Alexeev, ${ }^{32}$ G. Alkhazov, ${ }^{36}$ A. Alton,,${ }^{57 *}$ A. Askew,${ }^{44}$ S. Atkins, ${ }^{55}$ K. Augsten, ${ }^{7}$ C. Avila,${ }^{5}$ F. Badaud,${ }^{10}$ L. Bagby,${ }^{45}$ B. Baldin,${ }^{45}$ D. V. Bandurin, ${ }^{44}$ S. Banerjee ${ }^{26}$ E. Barberis, ${ }^{56}$ P. Baringer, ${ }^{53}$ J. F. Bartlett,${ }^{45}$ U. Bassler, ${ }^{15}$ V. Bazterra, ${ }^{46}$ A. Bean,${ }^{53}$ M. Begalli, ${ }^{2}$ L. Bellantoni, ${ }^{45}$ S. B. Beri, ${ }^{24}$ G. Bernardi, ${ }^{14}$ R. Bernhard, ${ }^{19}$ I. Bertram, ${ }^{39}$ M. Besançon, ${ }^{15}$ R. Beuselinck,${ }^{40}$ P. C. Bhat,${ }^{45}$ S. Bhatia, ${ }^{59}$ V. Bhatnagar, ${ }^{24}$ G. Blazey, ${ }^{47}$ S. Blessing, ${ }^{44}$ K. Bloom, ${ }^{60}$ A. Boehnlein, ${ }^{45}$ D. Boline,${ }^{65}$ E. E. Boos, ${ }^{34}$ G. Borissov, ${ }^{39}$ A. Brandt ${ }^{71}$ O. Brandt,${ }^{20}$ R. Brock, ${ }^{58}$ A. Bross, ${ }^{45}$ D. Brown, ${ }^{14}$ J. Brown, ${ }^{14}$ X. B. Bu, ${ }^{45}$ M. Buehler, ${ }^{45}$ V. Buescher, ${ }^{21}$ V. Bunichev, ${ }^{34}$ S. Burdin, ${ }^{39, \dagger}$ C. P. Buszello, ${ }^{38}$ E. Camacho-Pérez,${ }^{29}$ B. C. K. Casey, ${ }^{45}$ H. Castilla-Valdez, ${ }^{29}$ S. Caughron, ${ }^{58}$ S. Chakrabarti, ${ }^{65}$ D. Chakraborty, ${ }^{47}$ K. M. Chan, ${ }^{51}$ A. Chandra,${ }^{73}$ E. Chapon, ${ }^{15}$ G. Chen,${ }^{53}$ S. W. Cho, ${ }^{28}$ S. Choi, ${ }^{28}$

B. Choudhary, ${ }^{25}$ S. Cihangir, ${ }^{45}$ D. Claes,${ }^{60}$ J. Clutter, ${ }^{53}$ M. Cooke, ${ }^{45}$ W. E. Cooper, ${ }^{45}$ M. Corcoran, ${ }^{73}$ F. Couderc, ${ }^{15}$ M.-C. Cousinou, ${ }^{12}$ D. Cutts, ${ }^{70}$ A. Das,${ }^{42}$ G. Davies,${ }^{40}$ S. J. de Jong,${ }^{30,31}$ E. De La Cruz-Burelo,${ }^{29}$ F. Déliot,${ }^{15}$ R. Demina ${ }^{64}$ D. Denisov ${ }^{45}$ S. P. Denisov, ${ }^{35}$ S. Desai, ${ }^{45}$ C. Deterre, ${ }^{20,8}$ K. DeVaughan,${ }^{60}$ H. T. Diehl,${ }^{45}$ M. Diesburg,${ }^{45}$ P. F. Ding, ${ }^{41}$ A. Dominguez, ${ }^{60}$ A. Dubey, ${ }^{25}$ L. V. Dudko, ${ }^{34}$ D. Duggan, ${ }^{61}$ A. Duperrin, ${ }^{12}$ S. Dutt,${ }^{24}$ A. Dyshkant, ${ }^{47}$ M. Eads, ${ }^{47}$ D. Edmunds, ${ }^{58}$ J. Ellison, ${ }^{43}$ V. D. Elvira,${ }^{45}$ Y. Enari, ${ }^{14}$ H. Evans, ${ }^{49}$ V. N. Evdokimov, ${ }^{35}$ G. Facini, ${ }^{56}$ L. Feng, ${ }^{47}$ T. Ferbel, ${ }^{64}$ F. Fiedler, ${ }^{21}$ F. Filthaut, ${ }^{30,31}$ W. Fisher, ${ }^{58}$ H. E. Fisk, ${ }^{45}$ M. Fortner, ${ }^{47}$ H. Fox,${ }^{39}$ S. Fuess, ${ }^{45}$ A. Garcia-Bellido, ${ }^{64}$ J. A. García-González, ${ }^{29}$ G. A. García-Guerra, ${ }^{29, \ddagger}$ V. Gavrilov, ${ }^{33}$ W. Geng, ${ }^{12,58}$ C. E. Gerber, ${ }^{46}$ Y. Gershtein, ${ }^{61}$ G. Ginther, ${ }^{45,64}$ G. Golovanov, ${ }^{32}$ P. D. Grannis, ${ }^{65}$ S. Greder, ${ }^{16}$ H. Greenlee,${ }^{45}$ G. Grenier, ${ }^{17}$ Ph. Gris, ${ }^{10}$ J.-F. Grivaz, ${ }^{13}$ A. Grohsjean, ${ }^{15,8}$ S. Grünendahl, ${ }^{45}$ M. W. Grünewald ${ }^{27}$ T. Guillemin, ${ }^{13}$ G. Gutierrez, ${ }^{45}$ P. Gutierrez, ${ }^{68}$ J. Haley, ${ }^{56}$ L. Han, ${ }^{4}$ K. Harder ${ }^{41}$ A. Harel,${ }^{64}$ J. M. Hauptman, ${ }^{52}$ J. Hays,${ }^{40}$ T. Head ${ }^{41}$ T. Hebbeker ${ }^{18}$ D. Hedin,${ }^{47}$ H. Hegab, ${ }^{69}$ A. P. Heinson, ${ }^{43}$ U. Heintz, ${ }^{70}$ C. Hensel, ${ }^{20}$ I. Heredia-De La Cruz, ${ }^{29}$ K. Herner, ${ }^{57}$ G. Hesketh,${ }^{41,9 l}$ M. D. Hildreth,${ }^{51}$ R. Hirosky, ${ }^{74}$ T. Hoang, ${ }^{44}$ J. D. Hobbs, ${ }^{65}$ B. Hoeneisen, ${ }^{9}$ J. Hogan,${ }^{73}$ M. Hohlfeld, ${ }^{21}$ I. Howley, ${ }^{71}$ Z. Hubacek, ${ }^{7,15}$ V. Hynek, ${ }^{7}$ I. Iashvili, ${ }^{63}$ Y. Ilchenko, ${ }^{72}$ R. Illingworth, ${ }^{45}$ A. S. Ito ${ }^{45}$ S. Jabeen, ${ }^{70}$ M. Jaffré, ${ }^{13}$ A. Jayasinghe, ${ }^{68}$ M. S. Jeong, ${ }^{28}$ R. Jesik, ${ }^{40}$ P. Jiang, ${ }^{4}$ K. Johns, ${ }^{42}$ E. Johnson, ${ }^{58}$ M. Johnson, ${ }^{45}$ A. Jonckheere, ${ }^{45}$ P. Jonsson, ${ }^{40}$ J. Joshi, ${ }^{43}$ A. W. Jung, ${ }^{45}$ A. Juste, ${ }^{37}$ E. Kajfasz,${ }^{12}$

D. Karmanov, ${ }^{34}$ P. A. Kasper, ${ }^{45}$ I. Katsanos, ${ }^{60}$ R. Kehoe, ${ }^{72}$ S. Kermiche, ${ }^{12}$ N. Khalatyan, ${ }^{45}$ A. Khanov, ${ }^{69}$ A. Kharchilava, ${ }^{63}$ Y. N. Kharzheev, ${ }^{32}$ I. Kiselevich, ${ }^{33}$ J. M. Kohli, ${ }^{24}$ A. V. Kozelov, ${ }^{35}$ J. Kraus, ${ }^{59}$ A. Kumar, ${ }^{63}$ A. Kupco, ${ }^{8}$ T. Kurča, ${ }^{17}$ V. A. Kuzmin, ${ }^{34}$ S. Lammers, ${ }^{49}$ G. Landsberg, ${ }^{70}$ P. Lebrun,,${ }^{17}$ H. S. Lee, ${ }^{28}$ S. W. Lee, ${ }^{52}$ W. M. Lee,${ }^{44}$ X. Lei,${ }^{42}$ J. Lellouch ${ }^{14}$ D. Li, ${ }^{14}$ H. Li,${ }^{74}$ L. Li,${ }^{43}$ Q. Z. Li,${ }^{45}$ J. K. Lim, ${ }^{28}$ D. Lincoln,${ }^{45}$ J. Linnemann, ${ }^{58}$ V. V. Lipaev, ${ }^{35}$ R. Lipton, ${ }^{45}$ H. Liu, ${ }^{72}$ Y. Liu, ${ }^{4}$ A. Lobodenko, ${ }^{36}$ M. Lokajicek, ${ }^{8}$ R. Lopes de $\mathrm{Sa},{ }^{65}$ R. Luna-Garcia, ${ }^{29, * *}$ A. L. Lyon, ${ }^{45}$ A. K. A. Maciel, ${ }^{1}$ R. Magaña-Villalba, ${ }^{29}$ S. Malik, ${ }^{60}$ V.L. Malyshev ${ }^{32}$ Y. Maravin,${ }^{54}$ J. Martínez-Ortega, ${ }^{29}$ R. McCarthy, ${ }^{65}$

C. L. McGivern, ${ }^{41}$ M. M. Meijer, ${ }^{30,31}$ A. Melnitchouk, ${ }^{45}$ D. Menezes, ${ }^{47}$ P. G. Mercadante,${ }^{3}$ M. Merkin,${ }^{34}$ A. Meyer, ${ }^{18}$ J. Meyer ${ }^{20}{ }^{2}$ F. Miconi,${ }^{16}$ N. K. Mondal,${ }^{26}$ M. Mulhearn, ${ }^{74}$ E. Nagy,${ }^{12}$ M. Naimuddin, ${ }^{25}$ M. Narain, ${ }^{70}$ R. Nayyar, ${ }^{42}$ H. A. Neal, ${ }^{57}$ J. P. Negret, ${ }^{5}$ P. Neustroev,${ }^{36}$ H. T. Nguyen, ${ }^{74}$ T. Nunnemann, ${ }^{22}$ J. Orduna, ${ }^{73}$ N. Osman, ${ }^{12}$ J. Osta, ${ }^{51}$ M. Padilla, ${ }^{43}$ A. Pal,${ }^{71}$ N. Parashar, ${ }^{50}$ V. Parihar,${ }^{70}$ S. K. Park,${ }^{28}$ R. Partridge,${ }^{70, \|}$ N. Parua,${ }^{49}$ A. Patwa, ${ }^{66}$ B. Penning, ${ }^{45}$ M. Perfilov, ${ }^{34}$ Y. Peters,${ }^{20}$ K. Petridis,${ }^{41}$ G. Petrillo, ${ }^{64}$ P. Pétroff,,${ }^{13}$ M.-A. Pleier, ${ }^{66}$ P. L. M. Podesta-Lerma, ${ }^{29, \dagger \dagger}$ V. M. Podstavkov, ${ }^{45}$ A. V. Popov, ${ }^{35}$ M. Prewitt, ${ }^{73}$ D. Price, ${ }^{49}$ N. Prokopenko, ${ }^{35}$ J. Qian, ${ }^{57}$ A. Quadt, ${ }^{20}$ B. Quinn, ${ }^{59}$ M. S. Rangel, ${ }^{1}$ K. Ranjan, ${ }^{25}$ P. N. Ratoff,,${ }^{39}$ I. Razumov, ${ }^{35}$ P. Renkel,${ }^{72}$ I. Ripp-Baudot,${ }^{16}$ F. Rizatdinova, ${ }^{69}$ M. Rominsky, ${ }^{45}$ A. Ross,${ }^{39}$ C. Royon, ${ }^{15}$ P. Rubinov, ${ }^{45}$ R. Ruchti, ${ }^{51}$ G. Sajot,${ }^{11}$ P. Salcido,${ }^{47}$ A. Sánchez-Hernández, ${ }^{29}$ M. P. Sanders, ${ }^{22}$ A. S. Santos, ${ }^{1,+1}$ G. Savage, ${ }^{45}$ L. Sawyer, ${ }^{55}$ T. Scanlon, ${ }^{40}$ R. D. Schamberger ${ }^{65}$ Y. Scheglov, ${ }^{36}$ H. Schellman ${ }^{48}$ C. Schwanenberger, ${ }^{41}$ R. Schwienhorst,${ }^{58}$ J. Sekaric,${ }^{53}$ H. Severini, ${ }^{68}$ E. Shabalina,${ }^{20}$ V. Shary, ${ }^{15}$ S. Shaw,${ }^{58}$ A. A. Shchukin, ${ }^{35}$ R. K. Shivpuri, ${ }^{25}$ V. Simak, ${ }^{7}$ P. Skubic, ${ }^{68}$ P. Slattery ${ }^{64}$ D. Smirnov, ${ }^{51}$ K. J. Smith, ${ }^{63}$ G. R. Snow, ${ }^{60}$ J. Snow, ${ }^{67}$ S. Snyder, ${ }^{66}$ S. Söldner-Rembold, ${ }^{41}$ L. Sonnenschein, ${ }^{18}$ K. Soustruznik, ${ }^{6}$ J. Stark, ${ }^{11}$ D. A. Stoyanova, ${ }^{35}$ M. Strauss, ${ }^{68}$ L. Suter, ${ }^{41}$ P. Svoisky, ${ }^{68}$ M. Titov, ${ }^{15}$ V. V. Tokmenin,${ }^{32}$ Y.-T. Tsai, ${ }^{64}$ D. Tsybychev, ${ }^{65}$ B. Tuchming, ${ }^{15}$ C. Tully, ${ }^{62}$ L. Uvarov, ${ }^{36}$ S. Uvarov, ${ }^{36}$ S. Uzunyan,${ }^{47}$ R. Van Kooten, ${ }^{49}$ W. M. van Leeuwen,${ }^{30}$ N. Varelas, ${ }^{46}$ E. W. Varnes,${ }^{42}$ I. A. Vasilyev, ${ }^{35}$ P. Verdier,${ }^{17}$ A. Y. Verkheev, ${ }^{32}$ L. S. Vertogradov, ${ }^{32}$ M. Verzocchi, ${ }^{45}$ M. Vesterinen, ${ }^{41}$ D. Vilanova, ${ }^{15}$ P. Vokac, ${ }^{7}$ H. D. Wahl,${ }^{44}$ M. H. L. S. Wang,${ }^{45}$ J. Warchol, ${ }^{51}$ G. Watts, ${ }^{75}$ M. Wayne, ${ }^{51}$ J. Weichert,${ }^{21}$ L. Welty-Rieger, ${ }^{48}$ A. White, ${ }^{71}$ D. Wicke, ${ }^{23}$ M. R. J. Williams, ${ }^{39}$ G. W. Wilson, ${ }^{53}$ M. Wobisch ${ }^{55}$ D. R. Wood, ${ }^{56}$ T. R. Wyatt, ${ }^{41}$ Y. Xie, ${ }^{45}$ R. Yamada ${ }^{45}$ S. Yang, ${ }^{4}$ T. Yasuda, ${ }^{45}$ Y. A. Yatsunenko, ${ }^{32}$ W. Ye,${ }^{65}$ Z. Ye,${ }^{45}$ H. Yin, ${ }^{45}$ K. Yip, ${ }^{66}$ S. W. Youn,${ }^{45}$ J. M. Yu, ${ }^{57}$ J. Zennamo, ${ }^{63}$ T. G. Zhao, ${ }^{41}$ B. Zhou, ${ }^{57}$ J. Zhu, ${ }^{57}$ M. Zielinski, ${ }^{64}$ D. Zieminska, ${ }^{49}$ and L. Zivkovic ${ }^{14}$

(D0 Collaboration) 
${ }^{1}$ LAFEX, Centro Brasileiro de Pesquisas Físicas, Rio de Janeiro, Brazil

${ }^{2}$ Universidade do Estado do Rio de Janeiro, Rio de Janeiro, Brazil

${ }^{3}$ Universidade Federal do ABC, Santo André, Brazil

${ }^{4}$ University of Science and Technology of China, Hefei, People's Republic of China

${ }^{5}$ Universidad de los Andes, Bogotá, Colombia

${ }^{6}$ Charles University, Faculty of Mathematics and Physics, Center for Particle Physics, Prague, Czech Republic

${ }^{7}$ Czech Technical University in Prague, Prague, Czech Republic

${ }^{8}$ Center for Particle Physics, Institute of Physics, Academy of Sciences of the Czech Republic, Prague, Czech Republic

${ }^{9}$ Universidad San Francisco de Quito, Quito, Ecuador

${ }^{10}$ LPC, Université Blaise Pascal, CNRS/IN2P3, Clermont, France

${ }^{11}$ LPSC, Université Joseph Fourier Grenoble 1, CNRS/IN2P3, Institut National Polytechnique de Grenoble, Grenoble, France

${ }^{12}$ CPPM, Aix-Marseille Université, CNRS/IN2P3, Marseille, France

${ }^{13}$ LAL, Université Paris-Sud, CNRS/IN2P3, Orsay, France

${ }^{14}$ LPNHE, Universités Paris VI and VII, CNRS/IN2P3, Paris, France

${ }^{15}$ CEA, Irfu, SPP, Saclay, France

${ }^{16} I P H C$, Université de Strasbourg, CNRS/IN2P3, Strasbourg, France

${ }^{17}$ IPNL, Université Lyon 1, CNRS/IN2P3, Villeurbanne, France and Université de Lyon, Lyon, France

${ }^{18}$ III. Physikalisches Institut A, RWTH Aachen University, Aachen, Germany

${ }^{19}$ Physikalisches Institut, Universität Freiburg, Freiburg, Germany

${ }^{20}$ II. Physikalisches Institut, Georg-August-Universität Göttingen, Göttingen, Germany

${ }^{21}$ Institut für Physik, Universität Mainz, Mainz, Germany

${ }^{22}$ Ludwig-Maximilians-Universität München, München, Germany

${ }^{23}$ Fachbereich Physik, Bergische Universität Wuppertal, Wuppertal, Germany

${ }^{24}$ Panjab University, Chandigarh, India

${ }^{25}$ Delhi University, Delhi, India

${ }^{26}$ Tata Institute of Fundamental Research, Mumbai, India

${ }^{27}$ University College Dublin, Dublin, Ireland

${ }^{28}$ Korea Detector Laboratory, Korea University, Seoul, Korea

${ }^{29}$ CINVESTAV, Mexico City, Mexico

${ }^{30}$ Nikhef, Science Park, Amsterdam, The Netherlands

${ }^{31}$ Radboud University Nijmegen, Nijmegen, The Netherlands

${ }^{32}$ Joint Institute for Nuclear Research, Dubna, Russia

${ }^{33}$ Institute for Theoretical and Experimental Physics, Moscow, Russia

${ }^{34}$ Moscow State University, Moscow, Russia

${ }^{35}$ Institute for High Energy Physics, Protvino, Russia

${ }^{36}$ Petersburg Nuclear Physics Institute, St. Petersburg, Russia

${ }^{37}$ Institució Catalana de Recerca i Estudis Avançats (ICREA) and Institut de Física d'Altes Energies (IFAE), Barcelona, Spain

${ }^{38}$ Uppsala University, Uppsala, Sweden

${ }^{39}$ Lancaster University, Lancaster LA1 4YB, United Kingdom

${ }^{40}$ Imperial College London, London SW7 2AZ, United Kingdom

${ }^{41}$ The University of Manchester, Manchester M13 9PL, United Kingdom

${ }^{42}$ University of Arizona, Tucson, Arizona 85721, USA

${ }^{43}$ University of California Riverside, Riverside, California 92521, USA

${ }^{44}$ Florida State University, Tallahassee, Florida 32306, USA

${ }^{45}$ Fermi National Accelerator Laboratory, Batavia, Illinois 60510, USA

${ }^{46}$ University of Illinois at Chicago, Chicago, Illinois 60607, USA

${ }^{47}$ Northern Illinois University, DeKalb, Illinois 60115, USA

${ }^{48}$ Northwestern University, Evanston, Illinois 60208, USA

${ }^{49}$ Indiana University, Bloomington, Indiana 47405, USA

${ }^{50}$ Purdue University Calumet, Hammond, Indiana 46323, USA

${ }^{51}$ University of Notre Dame, Notre Dame, Indiana 46556, USA

${ }^{52}$ Iowa State University, Ames, Iowa 50011, USA

${ }^{53}$ University of Kansas, Lawrence, Kansas 66045, USA

${ }^{54}$ Kansas State University, Manhattan, Kansas 66506, USA

${ }^{55}$ Louisiana Tech University, Ruston, Louisiana 71272, USA

${ }^{56}$ Northeastern University, Boston, Massachusetts 02115, USA

${ }^{57}$ University of Michigan, Ann Arbor, Michigan 48109, USA

${ }^{58}$ Michigan State University, East Lansing, Michigan 48824, USA

${ }^{59}$ University of Mississippi, University, Mississippi 38677, USA

${ }^{60}$ University of Nebraska, Lincoln, Nebraska 68588, USA

${ }^{61}$ Rutgers University, Piscataway, New Jersey 08855, USA 


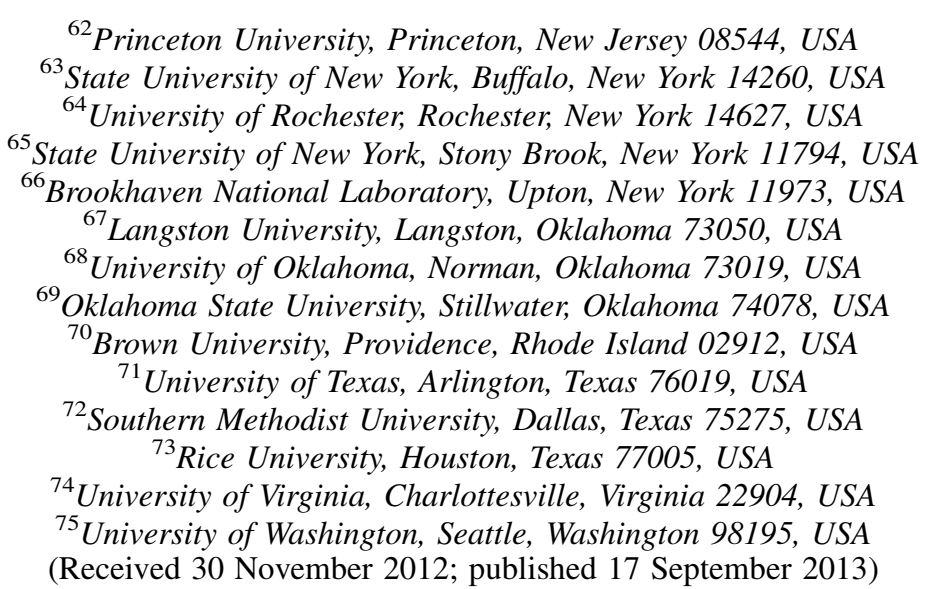

We present a search for the standard model Higgs boson in final states with an electron or muon and a hadronically decaying tau lepton in association with two or more jets using $9.7 \mathrm{fb}^{-1}$ of Run II Fermilab Tevatron Collider data collected with the D0 detector. The analysis is sensitive to Higgs boson production via gluon fusion, associated vector boson production, and vector boson fusion, followed by the Higgs boson decay to tau lepton pairs or to $W$ boson pairs. The ratios of $95 \%$ C.L. upper limits on the cross section times branching ratio to those predicted by the standard model are obtained for orthogonal subsamples that are enriched in either $H \rightarrow \tau \tau$ decays or $H \rightarrow W W$ decays, and for the combination of these subsample limits. The observed and expected limit ratios for the combined subsamples at a Higgs boson mass of $125 \mathrm{GeV}$ are 11.3 and 9.0 , respectively.

DOI: 10.1103/PhysRevD.88.052005

PACS numbers: $13.85 . \mathrm{Rm}$

\section{INTRODUCTION}

The standard model (SM) of particle physics postulates a complex Higgs doublet field as the source of electroweak symmetry breaking, giving rise to nonzero masses of the $W$ and $Z$ vector bosons and the fundamental fermions. The mass of the spin-zero Higgs boson, $H$, that survives after the symmetry breaking is not predicted by the SM, but is constrained by direct searches at the LEP [1], Tevatron [2] and LHC [3,4] colliders, and by precision electroweak measurements [5] to be in the range $122-127 \mathrm{GeV}$ at the 95\% C.L. The boson observed at a mass of about $126 \mathrm{GeV}$ by ATLAS and CMS $[3,4]$ when combining evidence for a narrow resonance in the $\gamma \gamma$ and $Z Z$ channels has production and decay properties that are consistent with the SM Higgs boson, given the current sensitivities. Evidence for a particle compatible with the discovered boson with the decay $b \bar{b}$ has been reported independently by the Tevatron experiments [6-8]. It is now important to measure its

\footnotetext{
*Visitor from Augustana College, Sioux Falls, SD, USA.

${ }^{\dagger}$ Visitor from The University of Liverpool, Liverpool, UK.

${ }^{\star}$ Visitor from UPIITA-IPN, Mexico City, Mexico.

${ }^{\S}$ Visitor from DESY, Hamburg, Germany.

"Visitor from SLAC, Menlo Park, CA, USA.

"Visitor from University College London, London, UK.

**Visitor from Centro de Investigacion en Computacion - IPN, Mexico City, Mexico.

${ }^{\dagger}$ Visitor from ECFM, Universidad Autonoma de Sinaloa, Culiacán, Mexico.

${ }^{\ddagger}$ Visitor from Universidade Estadual Paulista, São Paulo, Brazil.
}

couplings for all accessible particles, in particular to leptons for which no evidence currently exists.

In this paper we present a search for the Higgs boson in final states that are sensitive to both the $H \rightarrow \tau \tau$ and $H \rightarrow$ $W W$ decay modes containing a hadronically decaying tau lepton, a muon or electron plus at least two jets. We use data collected with the D0 detector at the Fermilab protonantiproton Tevatron Collider at $\sqrt{s}=1.96 \mathrm{TeV}$. The search is conducted at ten Higgs boson masses $\left(M_{H}\right)$ between 105 and $150 \mathrm{GeV}$ in $5 \mathrm{GeV}$ intervals. In the following $\tau_{h}$ represents a hadronically decaying tau lepton, $\ell$ denotes a lepton ( $\mu$ or $e$ ) and $j$ represents hadronic jets. The symbols " $\mu \tau j j$ " and " $e \tau j j$ " denote the two individual search channels, collectively described as " $\ell \tau j j$."

Over the mass range $115 \leq M_{H} \leq 150 \mathrm{GeV}$ the Higgs boson decay branching fractions vary considerably, with $H \rightarrow b \bar{b}$ being the dominant decay for $M_{H} \lesssim 135 \mathrm{GeV}$ and $H \rightarrow W^{+} W^{-}$becoming important for $M_{H} \gtrsim$ $135 \mathrm{GeV}$. Below $M_{H} \lesssim 125 \mathrm{GeV}, H \rightarrow \tau^{+} \tau^{-}$has an appreciable $(\approx 8 \%)$ branching fraction. Previous analyses by the D0 and CDF Collaborations have mainly focused on the decay modes $H \rightarrow b \bar{b}$ in the low mass region [6-8] and $H \rightarrow W W$ with both $W$ bosons decaying to a lepton and neutrino in the high mass region [2]. A D0 publication [9] reported a Higgs boson search in the $\tau_{h} \mu$ final state with zero or one jet, and the $\mu \tau j j$ and $e \tau j j$ final states, using $7.3,6.2$ and $4.3 \mathrm{fb}^{-1}$ of data, respectively. The CDF Collaboration has published a search in the $\tau_{h} \ell+\geq 1$ jet final state using $6.0 \mathrm{fb}^{-1}$ of data [10]. Here we report updated results for both the $\mu \tau j j$ and $e \tau j j$ searches with 
the full D0 $9.7 \mathrm{fb}^{-1} 2002-2011$ data set with an improved analysis using an extended set of variables for discriminating signal and background and an improved multivariate analysis. The current $\ell \tau j j$ analyses supersede those of Ref. [9].

The Higgs boson production processes considered are (i) gluon fusion (GF), $g g \rightarrow H$ ( + jets); (ii) vector boson fusion (VBF), $q \bar{q} \rightarrow q \bar{q} H$; (iii) associated vector boson and Higgs boson production (VH), $q \bar{q} \rightarrow V H$, where $V$ is a $W$ or $Z$ boson, and $V \rightarrow q \bar{q}$; and (iv) associated Higgs boson and $Z$ boson production (HZ), $q \bar{q} \rightarrow H Z$, with $H \rightarrow$ $b \bar{b}$ and $Z \rightarrow \tau \tau$. The GF, VBF, and $\mathrm{VH}$ processes are further subdivided according to the Higgs boson decay, $H \rightarrow \tau \tau$ or $H \rightarrow W W$, and these subchannels are denoted as $\mathrm{GF}_{\tau \tau}, \mathrm{GF}_{W W}$, etc.

Tau leptons can occur at lower $M_{H}$ through direct decays of the Higgs boson or, at higher $M_{H}$, indirectly from $H \rightarrow$ $V V$ with $V \rightarrow \tau+X$. The leptons may arise from $\tau$ decay or, at high $M_{H}$, directly from $V$ decay. Thus the $\ell \tau j j$ channels are more uniformly sensitive to Higgs boson production over the full mass range investigated than are the dedicated $H \rightarrow b \bar{b}$ or $H \rightarrow W W \rightarrow \ell \bar{\ell} \nu \bar{\nu}$ analyses, thus improving the combined search sensitivity, particularly in the intermediate mass region around $135 \mathrm{GeV}$.

\section{THE DO DETECTOR}

The D0 detector [11-13] contains tracking, calorimeter and muon subdetector systems. Silicon microstrip tracking detectors (SMT) near the interaction point cover pseudorapidity $|\eta|<3$ to provide tracking and vertexing information. The SMT $[14,15]$ contains cylindrical barrel layers aligned with their axes parallel to the beams and disk segments. The disks are perpendicular to the beam axis, interleaved with, and extending beyond, the barrels. The central fiber tracker (CFT) surrounds the SMT, providing coverage to about $|\eta|=2$. The CFT has eight concentric cylindrical layers of overlapped scintillating fibers providing axial and stereo $\left( \pm 3^{\circ}\right)$ measurements. A 1.9 T solenoid surrounds these tracking detectors.

Three uranium liquid-argon calorimeters measure particle energies. The central calorimeter (CC) covers $|\eta|<1$, and two end calorimeters (EC) extend coverage to about $|\eta|=4$. The calorimeter is highly segmented along the particle direction, with four electromagnetic (EM) and four or five hadronic sections in depth, and segmentation transverse to the particle direction with typically $\Delta \eta=\Delta \phi=0.1$, where $\phi$ is the azimuthal angle $(\Delta \eta=\Delta \phi=0.05$ in the third EM depth segment). The calorimeters are supplemented with central and forward scintillating strip preshower detectors (CPS and FPS) located in front of the CC and EC. Intercryostat detectors (ICD) provide added sampling in the region $1.1<|\eta|<1.4$ where the $\mathrm{CC}$ and $\mathrm{EC}$ cryostat walls degrade the calorimeter energy resolution.

Muons are measured just outside the calorimeters, and twice more outside $1.8 \mathrm{~T}$ iron toroidal magnets, over the range $|\eta|<2$. Each measurement is based on scintillation counters and several layers of tracking chambers. Scintillators surrounding the exiting beams allow determination of the luminosity [16].

A three-level trigger system selects events for data logging at about $100 \mathrm{~Hz}$. The first-level trigger (L1) is based on fast custom logic for several subdetectors and is capable of making decisions after each beam crossing. The secondlevel trigger (L2) makes microprocessor-based decisions using multidetector information. The third-level trigger (L3) uses fully digitized outputs from all detectors to refine the decision and select events for offline processing.

The data collected for this analysis come from the full Tevatron Run II period extending from 2002 to 2011. The data set is divided into two epochs, Runs IIa and IIb. Between these two epochs, substantial upgrades were made to the detector, including the addition of a new radiation-hard silicon strip detector close to the beam line and upgrades to the trigger system. The instantaneous luminosity of the Tevatron increased substantially between Run IIa and Run IIb. The integrated luminosities are 1.0 and $8.7 \mathrm{fb}^{-1}$ for the two epochs, respectively.

\section{TRIGGER}

The $\mu \tau j j$ data were collected using all triggers employed in D0. We reject events in which a muon candidate points toward the region of impaired coverage due to the detector supports. The trigger efficiency was determined in two steps. In the first step the efficiency for a suite of single muon triggers was measured using a tag and probe analysis of a sample of $Z \rightarrow \mu \mu$ events and found to be about $65 \%$ for that sample. In Run IIa the single muon triggers require the muon transverse momentum $p_{T}^{\mu}>12 \mathrm{GeV}$ and $\left|\eta_{d}^{\mu}\right|<$ 2.0, where $\eta_{d}^{\mu}$ is the pseudorapidity calculated assuming the muon originated at the center of the detector. Owing to the higher instantaneous luminosity in Run IIb, the trigger requirements were tightened to $p_{T}^{\mu}>15 \mathrm{GeV}$ and $\left|\eta_{d}^{\mu}\right|<$ 1.6. The single muon trigger efficiency is parametrized as a function of the $\eta_{d}$ and $\phi$ of the muon and instantaneous luminosity (as well as $p_{T}^{\mu}$ in Run IIa). The background and signal events simulated by Monte Carlo (MC) are weighted by these efficiency functions.

In the second step we measure the ratio $\mathcal{R}_{\text {all }}$ of the $\ell \tau j j$ signal sample data events collected with all triggers to those with single muon triggers, after subtracting the expected multijet component from both. (The signal and multijet samples are discussed in Secs. VI and VII.) We examine the dependences of this ratio upon the $p_{T}$ and $\eta$ of the $\mu, \tau_{h}$ and leading (highest $p_{T}$ ) jet. No significant dependences are observed, and a constant $\mathcal{R}_{\text {all }}$ is used as an additional weighting factor for the efficiency of the MC samples. The use of this inclusive trigger approach gives an increase in the data sample of about $40 \%$ compared to that from the single muon triggers alone. 
For the $e \tau j j$ analysis, we employ a set of triggers that require an EM object and a jet. The efficiency of the electron components of these triggers is obtained from a tag and probe analysis of $Z \rightarrow e e$ events and is parametrized in terms of electron $p_{T}$ and $\eta_{d}$. The efficiency of the jet component is measured in events selected by a single muon trigger in which a jet is reconstructed offline; the jet trigger term efficiency is then determined as a function of jet $p_{T}$ and $\eta_{d}$ on the basis of whether or not the corresponding muon plus jet trigger condition is satisfied. The impact of the correlation between electron and jet portions of the trigger is small. The trigger efficiency is about $85 \%$ for the signal processes.

\section{BACKGROUND AND SIGNAL SAMPLES}

The major backgrounds for the Higgs boson search are $Z$ and $W$ bosons produced in association with jets, $t \bar{t}$, and QCD multijet production (MJ) in which a jet simulates a lepton or hadronically decaying tau. Smaller backgrounds arise from boson ( $W, Z$ or $\gamma$ ) pair production ("diboson") and single top quark production which is included with the $t \bar{t}$ background. All but the MJ background are simulated using MC event generator programs and normalized to the highest order theoretical calculations available. These are referred to below as "SM" backgrounds. The MC simulations use the CTEQ6L1 parton distribution functions (PDF) [17].

The $Z+$ jets and $W+$ jets MC event samples are generated by ALPGEN [18], interfaced to PYTHIA [19] to provide initial and final state radiation and the hadronization of the produced partons. The $p_{T}^{Z}$ distribution is reweighted to agree with the D0 measurement [20]. The $p_{T}^{W}$ is also reweighted using the same experimental input, corrected for the theoretical differences between $W$ and $Z$ bosons expected in next-to-next-to-leading order (NNLO) QCD [21]. The $Z+$ jets and $W+$ jets cross sections are normalized using the calculations of Ref. [22] and the MSTW 2008 NNLO PDFs [23].

We simulate $t \bar{t}$ and single top quark events using the ALPGEN and SINGLETOP [24] generators, respectively, with the parton hadronization provided by PYTHIA. The normalizations are based on approximate NNLO QCD calculations [25]. The diboson events are generated by PYTHIA and normalized with MCFM [26].

Higgs boson production is simulated using PYTHIA, with normalizations taken from Ref. [2]. The Higgs boson decays are simulated using HDECAY [27], and the $\tau$ decays are obtained from TAUOLA [28].

The MC signal and background events are passed through the GEANT3-based [29] simulation of the detector response. Prior to reconstructing the $\mathrm{MC}$ events with the standard data programs, we superimpose events from a library of data events collected from random beam crossings to account for detector noise and pileup from additional $p \bar{p}$ collisions in the same or previous bunch crossings. The difference between the luminosity distribution for the random events and our data sample is encoded in a weight factor applied to the MC events. Simulated events are also weighted to account for the differences between MC and data for the lepton, tau, and jet identification efficiencies and for the energy scale and resolution of jets, in addition to the trigger weights discussed in Sec. III.

\section{OBJECT SELECTION CRITERIA}

Muon candidates are required to have hits in the muon chambers before and after the toroidal magnets, and to be matched to a track in the tracking system. Muons must be isolated from additional energy deposits in both the calorimeter and the tracking system. We require the calorimeter transverse energy $E_{T}^{\text {iso }}$ in the annular cone $0.1<$ $R<0.4$ around the muon to be less than $2.5 \mathrm{GeV}$, where $R=\sqrt{\Delta \eta^{2}+\Delta \phi^{2}}$, and we require that the sum of the transverse momenta of tracks within a cone $R<0.5$, excluding that of the candidate muon, be less than $2.5 \mathrm{GeV}$. We reject cosmic ray induced muon candidates by requiring that the time of arrival at the muon system scintillation counters is within $10 \mathrm{~ns}$ of that expected for collision products. The muon isolation selections are reversed for a special MJ control sample, as discussed in Sec. VII.

Electrons are identified using information from the EM and hadronic calorimeters, tracking detectors and the preshower detectors to form a combined electron identification variable, $\zeta$. The main component of $\zeta$ is a likelihood variable, $\mathcal{L}_{e}$, defined using eight individual variables: the $\chi^{2}$ for the transverse and longitudinal shower profile to conform to that expected for an EM shower; the fraction of calorimeter energy observed in the EM layers; the number of CPS strips hit; the $\chi^{2}$ of the track match to the calorimeter cluster centroid; the ratio of track momentum and calorimeter cluster energy; the number of tracks in a cone $R<0.05$ around the electron; the sum of the transverse momenta of tracks within $R<0.4$ of the candidate track; and the distance of closest approach of the track to the primary vertex (PV). The rms width of the calorimeter cluster and the isolation of the calorimeter cluster from nearby energy also contribute to the determination of $\zeta$. We require $\zeta$ to exceed a threshold that is different for electrons in the $\mathrm{CC}$ and EC. The identification efficiencies are parametrized in terms of $p_{T}, \eta_{d}$ and $\phi$. At $p_{T}=25 \mathrm{GeV}$, the efficiencies for $\mathrm{CC}$ electrons are about $83 \%$, and they are about $50 \%$ for EC electrons, as measured in $Z \rightarrow e e$ events. In addition, electron candidate tracks in the CC region are required to impinge upon a calorimeter module within the central $80 \%$ of its azimuthal range. The $\mathcal{L}_{e}$ variable requirement is modified for the $\mathrm{MJ}$ control sample described in Sec. VII.

We select three types of hadronically decaying tau leptons based on the number of tracks within a cone $R<0.3$ and the number of EM subclusters found in the calorimeter using a nearest neighbor algorithm. Type 1 requires one track and no EM subclusters. Type 2 requires one track and 
at least one EM subcluster. Type 3 requires at least two tracks with or without EM subclusters. Type 3 candidates with exactly two tracks of opposite charge sign, for which the tau charge is ambiguous, are rejected. The visible $\tau_{h}$ transverse energy $E_{T}^{\tau}$ is constructed from the track momenta, augmented with EM calorimeter information. We require the sum of the track momenta associated with the $\tau_{h}$ $\left(p_{T}^{\tau}\right)$ to exceed $(7,5,7) \mathrm{GeV}, E_{T}^{\tau}>(12.5,12.5,15) \mathrm{GeV}$, and $\left(p_{T}^{\tau} / E_{T}^{\tau}\right)>(0.65,0.5,0.5)$ for tau types $(1,2,3)$, respectively, and the highest $p_{T}$ track for type 3 taus must exceed $5 \mathrm{GeV}$. We construct a neural network, $\mathrm{NN}_{\tau}$ [30], based on energy deposition patterns and isolation criteria in the calorimeter and tracking systems for each tau type to discriminate a tau from a misidentified jet. Figure 1 shows illustrative $\mathrm{NN}_{\tau}$ distributions taken from a control data sample of $Z \rightarrow \tau \tau$ candidates. We require $\mathrm{NN}_{\tau}$ to be greater than $(0.92,0.90,0.91)$ for tau types $(1,2,3)$, respectively. For type 2 taus, we construct a second neural network, $\mathrm{NN}_{\tau / e}$, to differentiate taus from electrons. The distribution of $\mathrm{NN}_{\tau / e}$, taken from the $e \tau j j$ sample described in Sec. VI, is shown in Fig. 1(d). We select events with $\mathrm{NN}_{\tau / e>0.5}$.

Jets are reconstructed using an iterative midpoint cone algorithm [31] with a cone size $R=0.5$. We require at least two tracks associated with the jet that point to the PV (vertex confirmation) in Run IIb due to the higher multiplicity of collisions within a bunch crossing. Jet energies are corrected to the particle level for out-of-cone showering,
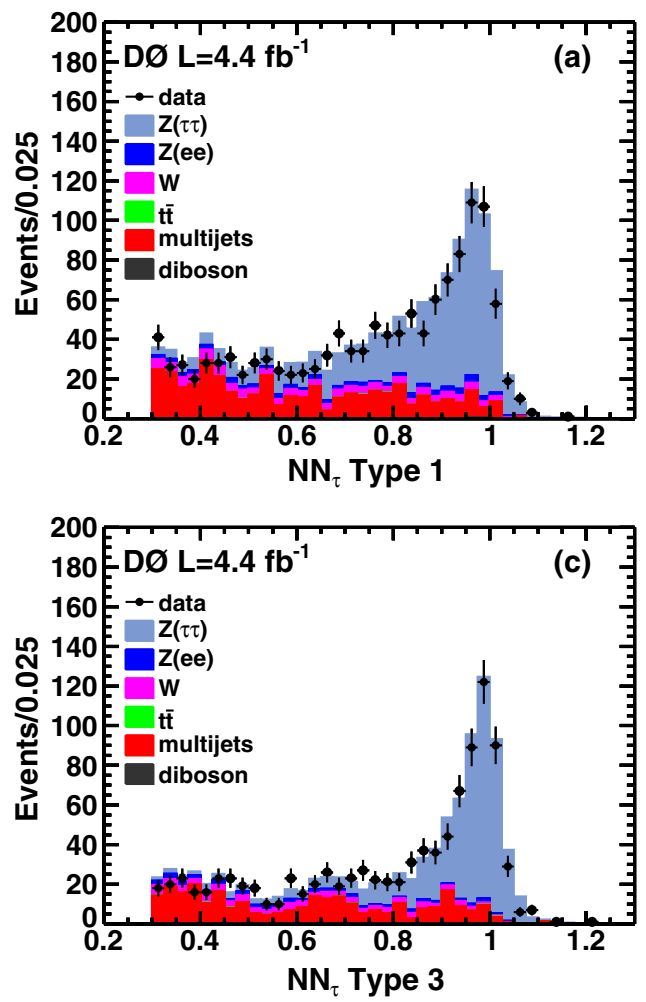

underlying event energy deposits and pileup from neighboring beam crossings, and for the effects of energy carried by muons and neutrinos when there is evidence for semileptonic decays of the jet particles. Jets in data are corrected for energy scale and resolution using $\gamma+$ jet and dijet samples. The MC jets are corrected for energy scale and resolution, as well as for the jet identification efficiency, to bring the MC responses into agreement with data. For the MC samples rich in quark jets (t $\bar{t}$ and diboson), there is an additional calibration applied to the jet energy that accounts for the differences between the responses of quark jets and the dominantly gluon jets for which the jet energy scale correction was obtained.

The missing transverse energy $\not{E}_{T}$ is computed from the observed transverse energy deposits in the calorimeter and is adjusted for the appropriate energy scale corrections for all objects, for isolated muons that deposit less than their full energy in the calorimeter, and for the unclustered energy in the calorimeters not associated with jets or EM objects. We define a quantity $\mathcal{S}$ that measures the significance of the $\mathscr{E}_{T}$ to be different from zero, based on the measured resolutions of the components of the $\mathbb{E}_{T}$ calculation [32].

\section{EVENT SELECTION CRITERIA}

We select a sample of events ("signal sample") with the criteria given below. Some of these differ for the Run IIa and Run IIb selection owing to the differences in the
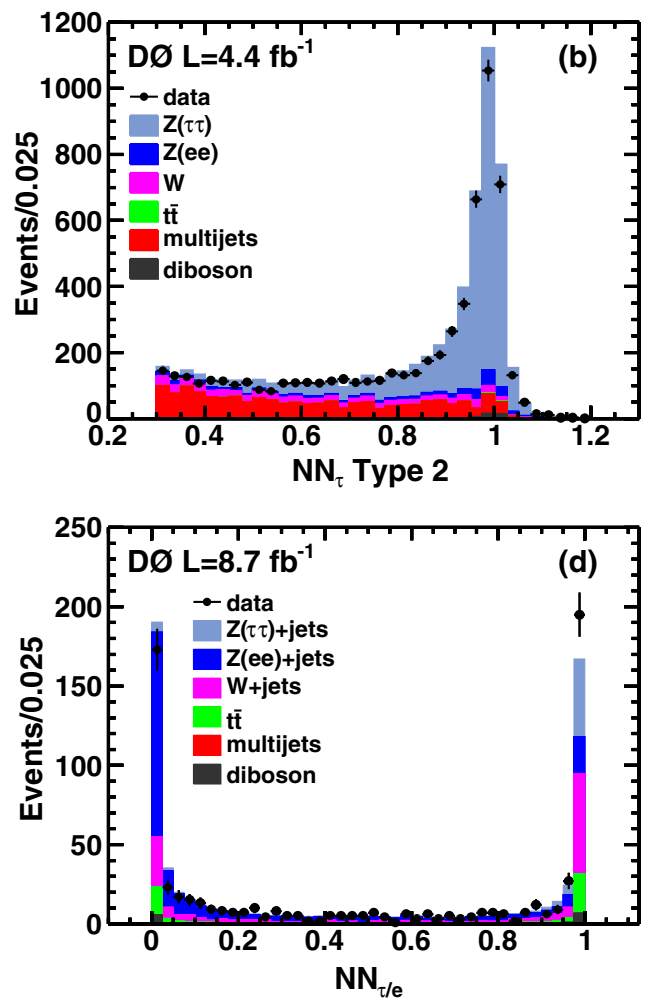

FIG. 1 (color online). The $\mathrm{NN}_{\tau}$ distribution for (a) type 1 taus, (b) type 2 taus, (c) type 3 taus, using data taken from a sample of $Z \rightarrow \tau \tau$ events with no requirements on the number of associated jets, and (d) the $\mathrm{NN}_{\tau / e}$ distribution for tau type 2 from a sample of $e \tau j j$ events. 
TABLE I. For each analysis channel, we give the number of background events expected from SM processes and MJ background, and those observed in data, for individual tau types and for the sum of all tau types after preselection. "Type" denotes $\tau$ type, " $V \mathrm{j}$ " denotes $W$ or $Z+$ jets and "DB" denotes diboson processes. The uncertainty on the sum of all backgrounds includes both MC statistical and systematic uncertainties.

\begin{tabular}{|c|c|c|c|c|c|c|c|c|}
\hline Type & $t \bar{t}$ & $W \mathrm{j}$ & $Z_{\ell \ell \mathrm{j}}$ & $Z_{\tau \tau} \mathrm{j}$ & DB & MJ & $\Sigma \mathrm{Bkd}$ & Data \\
\hline \multicolumn{9}{|c|}{$\mu \tau j j$ analysis } \\
\hline 1 & 15.3 & 10.2 & 4.4 & 37.1 & 2.3 & 39.1 & $108.4 \pm 7.4$ & 119 \\
\hline 2 & 121.3 & 65.2 & 29.3 & 241.8 & 14.5 & 135.4 & $607.5 \pm 47.1$ & 684 \\
\hline 3 & 20.2 & 39.1 & 4.4 & 54.5 & 3.2 & 50.6 & $172.1 \pm 12.4$ & 187 \\
\hline All & 156.9 & 114.5 & 38.1 & 333.4 & 16.0 & 225.1 & $888.0 \pm 49.2$ & 990 \\
\hline \multicolumn{9}{|c|}{$e \tau j j$ analysis } \\
\hline 1 & 4.5 & 4.6 & 0.0 & 9.8 & 0.9 & 3.1 & $23.0 \pm 1.8$ & 15 \\
\hline 2 & 57.7 & 64.9 & 66.6 & 91.7 & 8.3 & 1.7 & $290.8 \pm 21.0$ & 261 \\
\hline 3 & 27.2 & 47.2 & 2.4 & 28.5 & 3.7 & 14.6 & $123.7 \pm 9.6$ & 124 \\
\hline All & 89.4 & 116.7 & 69.1 & 130.0 & 12.9 & 19.4 & $437.5 \pm 23.2$ & 400 \\
\hline
\end{tabular}

detector, triggers and luminosity, and the fact that the jet vertex confirmation was not applied in Run IIa, leading to differences in the modeling of jet related variables.

For the $\mu \tau j j$ selection we require the following:

(i) A muon as defined in Sec. V with $p_{T}^{\mu}>12(15) \mathrm{GeV}$ and $\left|\eta_{d}^{\mu}\right|<2.0(1.6)$, for Run IIa (Run IIb).

(ii) At least one hadronic tau as defined in Sec. V with $\left|\eta_{d}^{\tau}\right| \leq 2$. The tau candidate with the highest $p_{T}$ is chosen for the analysis, and must have a charge sign that is opposite to the muon.

(iii) Two jets with $\left|\eta_{d}^{\text {jet }}\right|<3.4$; the leading jet ("jet1") is required to have $p_{T}^{\text {jetl }}>20 \mathrm{GeV}$ and a secondleading jet ("jet2") to have $p_{T}^{\text {jet2 }}>15 \mathrm{GeV}$.

(iv) No other electron with $p_{T}^{e}>10 \mathrm{GeV}$ and no other muon with $p_{T}^{\mu}>10 \mathrm{GeV}$ to retain orthogonality to other D0 searches for the SM Higgs boson.

(v) The scalar sum of all jet $p_{T}$ 's in the event $\left(H_{T}\right)$ must be greater than $80 \mathrm{GeV}$ for Run IIa to improve the modeling of jet related variables.
For the $e \tau j j$ selection we require the following:

(i) An electron as defined in Sec. V with $p_{T}^{e}>15 \mathrm{GeV}$, and $\left|\eta_{e}\right|<1.1$ or $1.5<\left|\eta_{e}\right|<2.5$.

(ii) At least one hadronic tau as defined in Sec. V with $\left|\eta_{d}^{\tau}\right| \leq 2$. The tau with the highest $p_{T}$ is chosen for the analysis and must have a charge sign that is opposite to the electron.

(iii) No type 1 taus with $1.05<|\eta|<1.5$, and type 2 taus must have $\mathrm{NN}_{\tau / e>0.5}$, to reduce the $Z(e e)+$ jets background in which an electron is misidentified as a tau.

(iv) Two jets with $\left|\eta_{d}^{\text {jet }}\right|<3.4 ; p_{T}^{\text {jet1 }}>25(20) \mathrm{GeV}$ for Run IIa (Run IIb) and $p_{T}^{\text {jet2 }}>15 \mathrm{GeV}$.

(v) No other electron with $p_{T}^{e}>12.5 \mathrm{GeV}$ and no muon with $p_{T}^{\mu}>12 \mathrm{GeV}$ and $\left|\eta_{d}^{\mu}\right|<2.0$ for orthogonality to other D0 SM Higgs boson searches.

(vi) The $\mathscr{E}_{T}$ significance variable is required to be $\mathcal{S}>3$ (2) for Run IIa (Run IIb) to reduce the MJ and $Z \rightarrow e e$ backgrounds.

TABLE II. For each analysis channel, we give the number of signal events expected for each of the nine production and decay processes, prior to the separation into the $\mathrm{T}$ and $\mathrm{W}$ subsamples discussed in the text.

\begin{tabular}{ccccccccccc}
\hline \hline$M_{H}$ & $\mathrm{HZ}$ & $\mathrm{ZH}_{\tau \tau}$ & $\mathrm{WH}_{\tau \tau}$ & $\mathrm{GF}_{\tau \tau}$ & $\mathrm{VBF}_{\tau \tau}$ & $\mathrm{ZH}_{W W}$ & $\mathrm{WH}_{W W}$ & $\mathrm{GF}_{W W}$ & $\mathrm{VBF}_{W W}$ & Total \\
\hline & & & \multicolumn{7}{c}{$\mu \tau j j$ analysis } \\
\hline 105 & 0.19 & 0.47 & 0.71 & 0.66 & 0.37 & 0.03 & 0.04 & 0.10 & 0.01 & 2.58 \\
115 & 0.15 & 0.38 & 0.57 & 0.53 & 0.34 & 0.09 & 0.15 & 0.08 & 0.05 & 2.34 \\
125 & 0.10 & 0.27 & 0.40 & 0.45 & 0.26 & 0.19 & 0.37 & 0.18 & 0.14 & 2.35 \\
135 & 0.06 & 0.16 & 0.23 & 0.30 & 0.18 & 0.33 & 0.63 & 0.31 & 0.24 & 2.43 \\
145 & 0.03 & 0.08 & 0.11 & 0.17 & 0.10 & 0.47 & 0.81 & 0.59 & 0.37 & 2.72 \\
& & & & & $e \tau j j$ analysis & & & & \\
105 & 0.14 & 0.34 & 0.53 & 0.32 & 0.26 & 0.01 & 0.01 & 0.02 & 0.00 & 1.63 \\
115 & 0.11 & 0.31 & 0.47 & 0.32 & 0.24 & 0.04 & 0.05 & 0.01 & 0.01 & 1.56 \\
125 & 0.07 & 0.22 & 0.33 & 0.30 & 0.21 & 0.09 & 0.14 & 0.04 & 0.04 & 1.46 \\
135 & 0.05 & 0.15 & 0.21 & 0.21 & 0.15 & 0.17 & 0.26 & 0.11 & 0.08 & 1.38 \\
145 & 0.02 & 0.07 & 0.11 & 0.12 & 0.08 & 0.25 & 0.35 & 0.16 & 0.11 & 1.26 \\
\hline \hline
\end{tabular}


Events with the same selection criteria, except that the $\ell$ and $\tau_{h}$ have the same charge sign, are retained for estimating the MJ background in both the $\mu \tau j j$ and $e \tau j j$ analyses (SS signal samples).

\section{MULTIJET BACKGROUND}

The MJ background arising from misidentification of leptons or taus by the detector reconstruction algorithms is difficult to simulate, so it is estimated using data. We define a sample of MJ-enriched events (MJ control sample, $\mathcal{M}$ ) which is large compared with the size of the signal sample. We use the events in $\mathcal{M}$, after subtraction of the small residual SM backgrounds simulated by MC, to provide the shapes of the MJ background kinematic distributions. We also use the $\mathcal{M}$ sample to obtain the MJ background normalization. For each tau type, the SM subtracted sample $\mathcal{M}$ is divided into the opposite sign $\ell \tau_{h}$ (OS) and same sign $\ell \tau_{h}$ (SS) samples. The ratio of OS and SS events in $\mathcal{M}$ is used to scale the number of MJ events in the SS signal sample to obtain the normalization for the MJ background in the signal sample. The MJ yield in the SS signal sample is obtained after subtracting the small SM backgrounds. Denoting the number of events in $\mathcal{M}$ by $M$ and the number of events in the SS or OS signal samples by $N$, the method is expressed by

$$
N_{\mathrm{OS}}^{\mathrm{MJ}}=\rho\left(N_{\mathrm{SS}}^{\mathrm{data}}-N_{\mathrm{SS}}^{\mathrm{SM}}\right)
$$

with

$$
\rho=\left(M_{\mathrm{OS}}^{\mathrm{data}}-M_{\mathrm{OS}}^{\mathrm{SM}}\right) /\left(M_{\mathrm{SS}}^{\mathrm{data}}-M_{\mathrm{SS}}^{\mathrm{SM}}\right) .
$$

This background estimate is computed separately for each tau type and summed to give the total MJ background.

For the $\mu \tau j j$ analysis, the MJ sample $\mathcal{M}$ is obtained by reversing at least one of the muon isolation requirements. The tau selection requirements are the same as for the signal sample. The MJ purity in this sample is about 97\%. We observe no significant dependence of the $\rho$ factors on the $p_{T}$ or $\eta$ of $\mu, \tau_{h}$ or jets, and therefore take them to be constant. Their values are within about $15 \%$ of unity and are similar for the three tau types, and in Run IIa and Run IIb. We observe that the modeling of the shapes of variables which employ the jet $p_{T}$ 's needs improvement and so we adopt a reweighting for the MJ events based upon the comparison of the distribution shapes in the SS signal sample and the MJ control sample. We find that the simple reweighting function $A e^{-B H_{T}}$, fitted to the ratio of the $H_{T}$ distributions for the SS signal and MJ control samples, gives adequate modeling.

For the $e \tau j j$ analysis, $\mathcal{M}$ is obtained by requiring the tau selection $0.3<\mathrm{NN}_{\tau}<0.9$, and by placing an upper bound of 0.85 on the $\mathcal{L}_{e}$ in the electron identification. In forming $\mathcal{M}$, no requirement is made on the $\mathbb{E}_{T}$ significance $\mathcal{S}$. For the Run IIb data, the cut on $\mathcal{S}$ in the signal selection leaves a negligibly small MJ contribution in the SS signal sample after the SM background subtraction. Owing to the absence
TABLE III. Variables used for $\mu \tau j j$ and $e \tau j j$ analyses in BDT training. As indicated, three variables are used for only one analysis.

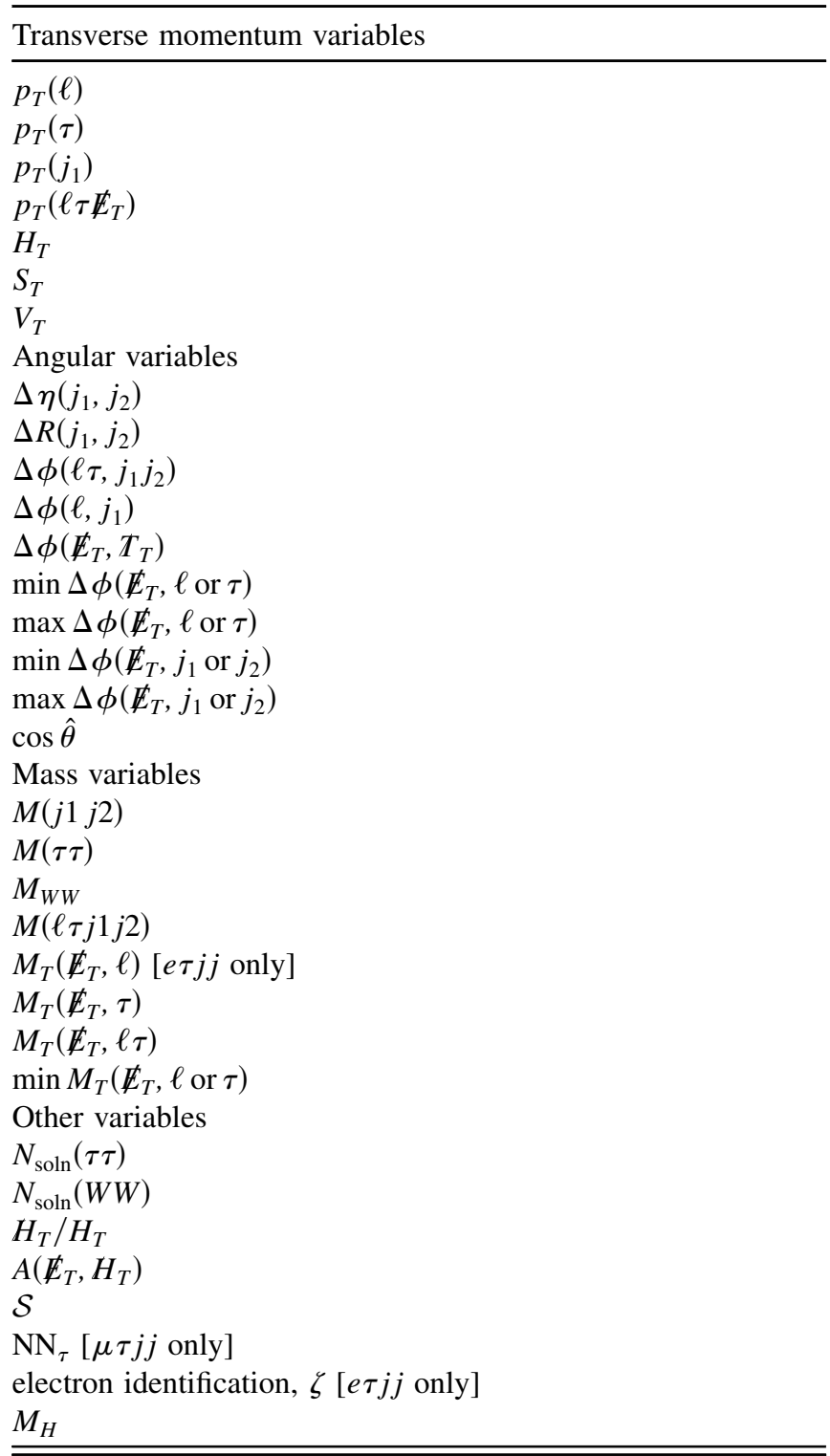

of jet vertex confirmation, some MJ background remains in Run IIa and the procedure used in the $\mu \tau j j$ analysis for its estimation is followed, except that no $H_{T}$ reweighting is needed.

For Run IIa, the MJ purity in $\mathcal{M}$ is $98 \%$ and the $\rho$-values are about 1.25.

\section{EVENT YIELDS}

The numbers of data and expected background events are given in Table I for the $\mu \tau j j$ and $e \tau j j$ analyses. Taking into account the systematic uncertainties on the backgrounds (see Sec. X) and uncertainties on the MC statistics, the predicted backgrounds are in agreement with the observed yields. The $e \tau j j$ yields are smaller than those for 
$\mu \tau j j$ primarily because of the requirements made for the $e \tau j j$ analysis on $\mathcal{S}, \eta_{\tau}, \phi_{e}$ in the $\mathrm{CC}$ region, and $\mathrm{NN}_{\tau / e}$ to reduce the $\mathrm{MJ}$ and $Z \rightarrow e e$ backgrounds. Representative expected signal yields for the nine production and decay processes are given in Table II.

\section{MULTIVARIATE ANALYSIS}

The number of background events greatly exceeds the expected number of Higgs boson signal events so we employ multivariate techniques that discriminate signal from background by taking into account the correlations among the variables. The multivariate strategy for this analysis is complicated by the presence of many distinct signals of comparable size, each with its own characteristic kinematic properties.

We tested several artificial learning techniques including neural networks and decision trees using the TMVA suite of programs [33] to implement the multivariate methods. We find an optimum performance with the gradient boosted decision tree classifier (BDT) which offers the advantage over neural networks that the use of variables that do not discriminate significantly between a particular signal and background, or are highly correlated with other variables, does not compromise the classifier performance. In the BDT $[34,35]$ approach, a series of splittings of the event sample is made at a sequential set of nodes into background-like and signal-like subsample nodes. The splitting is based upon the purity of signal and background events in a given node $N$ and its signal-like and background-like daughter nodes $S$ and $B$. Purity is defined as $p=s /(s+b)$, where $s(b)$ is the number of signal (background) events in the node. In the training, the event category is known either from the $\mathrm{MC}$ generation or $\mathrm{MJ}$ control samples. The optimum splitting is achieved by maximizing the decrease of the Gini index [36] $i=2 p(1-p)=$ $2 s b /(s+b)^{2}$ going from the parent node $N$ to the two daughter nodes $S$ and $B$, considering all choices of input variable and cut thresholds for that variable. Each such subsample is subjected to further splitting until the sample sizes reach a preset value. At each node, a random sampling of events is chosen from the full sample to help mitigate the effects of finite statistics. The training is recursive, with misclassified events in one cycle being reweighted for the next cycle. The TMVA training is controlled by parameters such as the maximum number of trees, the degree of reweighting in successive cycles, the fraction of the full sample used at each node and the number of cycles allowed. We varied these parameters around their nominal settings to obtain optimum values for our analysis.

\section{A. BDT variables}

We examine a large set of potentially discriminating kinematic variables with which to train the multivariate analyses. We choose a subset of well-modeled variables for which the agreement of data with expected background is good and which discriminate between at least one individual signal and background. In these distributions the expected signal contribution to the data is small. Table III shows the variables used for both the $\mu \tau j j$ and $e \tau j j$ analyses.

In Table III $H_{T}$ is the scalar sum of all jets with $p_{T}>$ $15 \mathrm{GeV}, S_{T}=H_{T}+\left|p_{T}^{\ell}\right|+\left|p_{T}^{\tau}\right|$, and $V_{T}$ is the magnitude
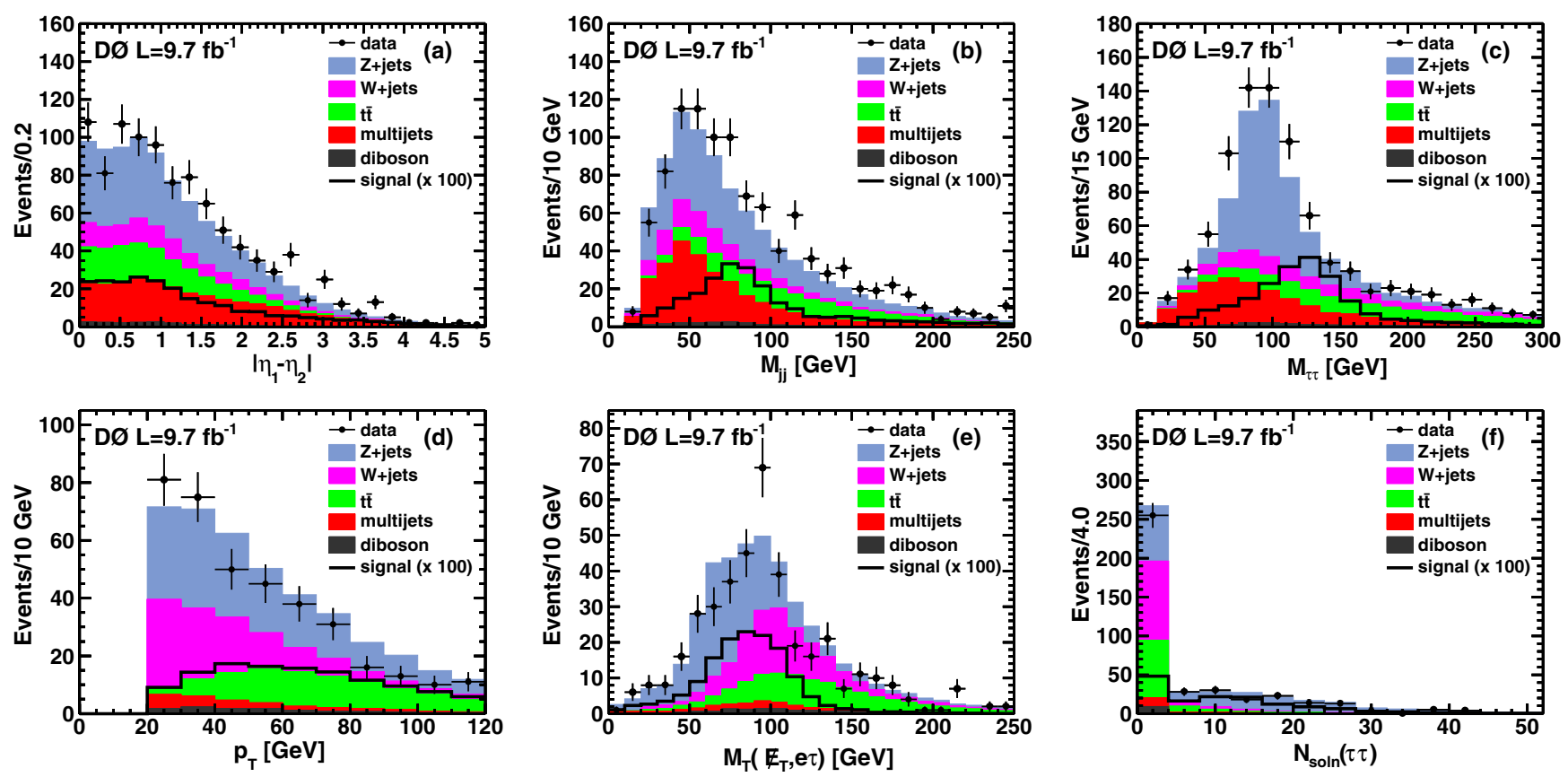

FIG. 2 (color online). Input variables for the $\mu \tau j j$ BDT training, (a) $\Delta \eta\left(j_{1}, j_{2}\right)$, (b) $M(j 1 j 2)$, (c) $M(\tau \tau)$, and for the $e \tau j j$ BDT training, (d) $p_{T}\left(j_{1}\right)$, (e) $M_{T}\left(\mathscr{E}_{T}, \ell \tau\right)$, (f) $N_{\text {soln }}(\tau \tau)$. 

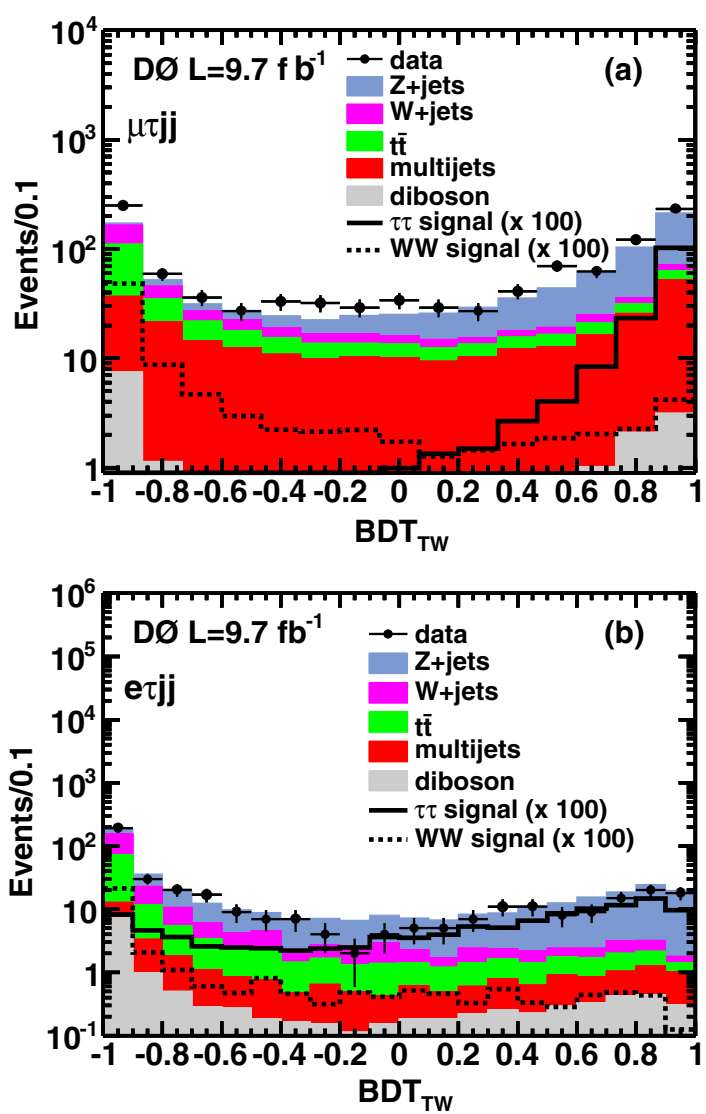

FIG. 3 (color online). BDT $_{\mathrm{TW}}$ outputs from the training of $H \rightarrow \tau \tau$ vs. $H \rightarrow W W$ for (a) the $\mu \tau j j$ analysis and (b) the $e \tau j j$ analysis. The solid (dashed) lines show the sum of all production processes with $H \rightarrow \tau \tau(H \rightarrow W W)$ decays at $M_{H}=$ $125 \mathrm{GeV}$. Events near $\mathrm{BDT}_{\mathrm{TW}}=-1$ are dominantly $H \rightarrow W W$ and those near +1 are mainly $H \rightarrow \tau \tau$.

of the vector transverse momenta of $\ell, \tau_{h}$ and all jets. The variable $H_{T}$ is the magnitude of the vectorial sum of all jet transverse momenta, and $T_{T}$ is the magnitude of the vector sum of all tracks emanating from the primary vertex. The variable $A\left(\not_{T}, H_{T}\right)$ is the ratio of the difference and the sum of $\mathscr{E}_{T}$ and $H_{T}$. Variables $\Delta \phi(a, b)$ are the difference in azimuthal angle between object $a$ and the object, or pair of objects, $b$, and similarly for the $\Delta \eta$ and $\Delta R$ variables. The variable $\hat{\theta}$ is the angle between the dijet system and the proton beam direction in the laboratory frame. Variables $M(a b \ldots c)$ are invariant masses of objects $a, b, \ldots c$ and $M_{T}\left(\mathscr{E}_{T}, a\right)$ is the transverse mass computed from $M_{T}^{2}=$ $2 E_{T}^{a} \mathbb{E}_{T}(1-\cos \phi)$, where $\phi$ is the azimuthal angle between $\mathbb{E}_{T}$ and the object, or pair of objects, $a$.

The $M(\tau \tau)$ and $M(W W)$ variables are determined using the Missing Mass Calculator (MMC) method [37]. These masses are under-constrained owing to the neutrinos from $\tau$ or $W$ decay. We compute the most likely $\tau \tau$ mass by scanning over a grid in the three-dimensional space of the azimuthal angle separations of the visible $\tau$ decay products and the neutrino(s) for each $\tau$, and the invariant mass of the multiple neutrinos from one of the $\tau$ 's (e.g. $\tau \rightarrow \ell \nu \bar{\nu}$ ),
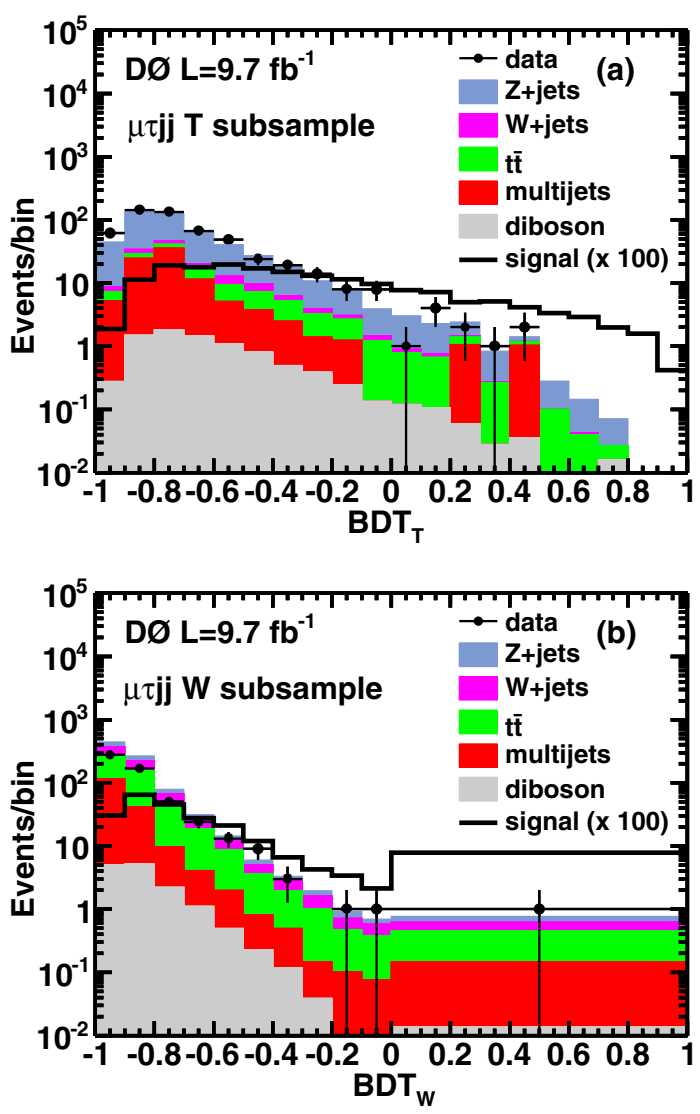

FIG. 4 (color online). Final discriminant distributions for the $\mu \tau j j$ analysis for (a) the $\mathrm{T}$ subsample at $M_{H}=125 \mathrm{GeV}$ and (b) the W subsample at $M_{H}=145 \mathrm{GeV}$.

given the constraints from the measured momenta of the visible decay products and the known $\tau$ mass. At each grid point, the calculated $\tau \tau$ mass is weighted by the probability for finding the $\Delta R$ at that point between the visible particles and the neutrinos. For $H \rightarrow W W$ in the mass region considered, one $W$ is virtual. The MMC mass is calculated for the $W W$ system, taking the mass of the virtual $W$ to be the most likely value $(38 \mathrm{GeV})$ for $M_{H}=125 \mathrm{GeV}$. The variables $N_{\text {soln }}(\tau \tau)$ and $N_{\text {soln }}(W W)$ are the number of physical mass solutions found in the grid search. Figure 2 shows the data and background distributions of representative BDT input variables.

One additional variable, the mass of the hypothesized Higgs boson, $M_{H}$, is used in training the BDTs as discussed below in Sec. IX C.

\section{B. Separation of $T$ and $W$ subsamples}

We perform a separation of the data, SM MC backgrounds and MJ background into two subsamples: one constructed to be rich in $H \rightarrow \tau \tau$ signals (the T subsample), and another rich in $H \rightarrow W W$ signals (the W subsample). We perform this separation into orthogonal data sets with a BDT $\left(\mathrm{BDT}_{\mathrm{TW}}\right)$ based on the variables listed in Table III using a TMVA training between the $H \rightarrow \tau \tau$ and $H \rightarrow W W$ 

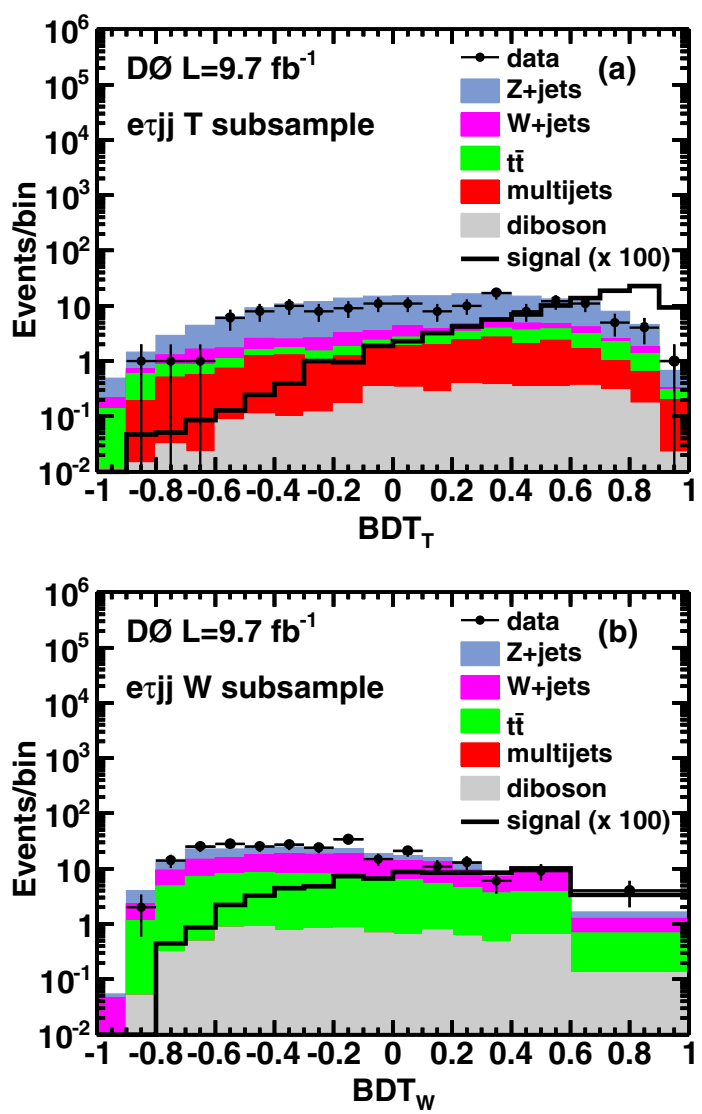

FIG. 5 (color online). Final discriminant distributions for the $e \tau j j$ analysis for (a) the $\mathrm{T}$ subsample at $M_{H}=125 \mathrm{GeV}$ and (b) the W subsample at $M_{H}=145 \mathrm{GeV}$.

MC signal events. Each subsample subsequently undergoes its own multivariate analysis and the results are combined at the limit-setting stage. The subsample separation gives about 15\%-20\% improvement in final Higgs boson limits over the no-separation case. It also provides an analysis for a purified $H \rightarrow \tau \tau$ signal, thus giving additional information on fermionic decays of the Higgs boson. We employ the $\mathrm{BDT}_{\mathrm{TW}}$ only to define the T and $\mathrm{W}$ subsamples; no further use of this variable is made. The $\mathrm{BDT}_{\mathrm{TW}}$ distributions for $\mu \tau j j$ and $e \tau j j$ are shown in Fig. 3.

We impose a cut to separate the $\mathrm{T}$ and $\mathrm{W}$ subsamples at $\mathrm{BDT}_{\mathrm{TW}}=+0.3$ for the $\mu \tau j j$ analysis and at -0.6 for the $e \tau j j$ analysis. The purity of $\tau \tau$ decays in the T subsample and of $W W$ decays in the $\mathrm{W}$ subsample is about $90 \%$ in the regions where the respective signals dominate. The dominant backgrounds in the $\mathrm{T}$ subsample are $Z+$ jets and (for the $\mu \tau j j$ analysis only) MJ. For the $\mathrm{W}$ subsample, the dominant backgrounds are $\mathrm{MJ}, t \bar{t}, W+$ jets and (for the $e \tau j j$ analysis only) $Z(e e)+$ jets.

\section{Global BDTs}

In searches for the Higgs boson, separate multivariate analyses have generally been performed for each Higgs boson mass under consideration. Due to variations in the details in the BDT training, this can lead to fluctuations in limits from one mass point to another. We have constructed a method that reduces such unwanted fluctuations.

We first perform a single training using the Higgs boson signal MCs at all mass points ("global BDT"). In this training, each signal event is characterized by the set of variables given in Table III, including the value of $M_{H}$ appropriate to each specific signal sample. To prevent the classifier from artificially separating backgrounds from signals based on the $M_{H}$ value, we randomly assign a $M_{H}$ value to the backgrounds with a $M_{H}$ distribution constructed to reproduce that of the signal samples. This assignment is done separately for the T and $\mathrm{W}$ subsamples and takes into account that there is an admixture of $H \rightarrow \tau \tau$ and $H \rightarrow W W$ decays in both cases. The $M_{H}$ variable does not play a strong role in separating signals from backgrounds in the global BDT training.

The global BDT is characterized by a set of training weights for the splitting of signal and backgrounds at each of the nodes of the tree. We then form the final discriminant at each $M_{H}$ by passing the MC signal events for a particular $M_{H}$ through the weights provided by the global BDT, now with $M_{H}$ set to the value under consideration. In this pass, the backgrounds are also provided with this specific $M_{H}$

TABLE IV. The range of systematic uncertainties (in percent) from different sources. Type N (S) denotes normalization (shape) uncertainties on the final discriminant. "XS" denotes "cross section." The "jets" uncertainties represent the independent uncertainties arising from jet vertex confirmation, jet identification and efficiency, jet energy resolution and jet energy scale, which, for a given channel and subsample, are similar.

\begin{tabular}{lcc}
\hline \hline Source & Type & Uncertainty (\%) \\
\hline Luminosity & $\mathrm{N}$ & 6.1 \\
$\mu$ ID/track match/isolation & $\mathrm{N}$ & 2.9 \\
$e$ ID/isolation & $\mathrm{N}$ & 4.0 \\
Single $\mu$ trigger efficiency & $\mathrm{N}$ & 5 \\
All trigger/single $\mu$ trigger & $\mathrm{N}$ & 7 \\
$e+$ Jets trigger efficiency & $\mathrm{N}$ & 2 \\
$\tau_{h}$ selection (by type) & $\mathrm{N}$ & $5.5 / 4.0 / 6.0$ \\
$\tau_{h}$ energy scale & $\mathrm{N}$ & 9.8 \\
$\tau_{h}$ track efficiency & $\mathrm{N}$ & 1.4 \\
$W / Z+$ jets XS & $\mathrm{N}$ & 6.0 \\
$t \bar{t}$, single top XS & $\mathrm{N}$ & 7.0 \\
Diboson XS & $\mathrm{N}$ & 6.0 \\
VH signal XS & $\mathrm{N}$ & 6.2 \\
VBF signal XS & $\mathrm{N}$ & 4.9 \\
GF signal XS normalization & $\mathrm{N}$ & 33 \\
GF signal XS PDF & $\mathrm{N}$ & 29 \\
Jets $\mu \tau j j$ T (W) subsample & $\mathrm{S}$ & $2-11(1-11)$ \\
Jets $e \tau j j$ T (W) subsample & $\mathrm{S}$ & $4-20(2-15)$ \\
PDF (signals) & $\mathrm{N}$ & 1.6 \\
PDF (backgrounds) & $\mathrm{N}$ & 2.0 \\
$\mu \tau j j$ MJ normalization & $\mathrm{N}$ & 5.3 \\
$e \tau j j$ MJ normalization & $\mathrm{N}$ & 5.0 \\
$\mu \tau j j$ MJ shape & $\mathrm{S}$ & $5-10$ \\
\hline \hline
\end{tabular}



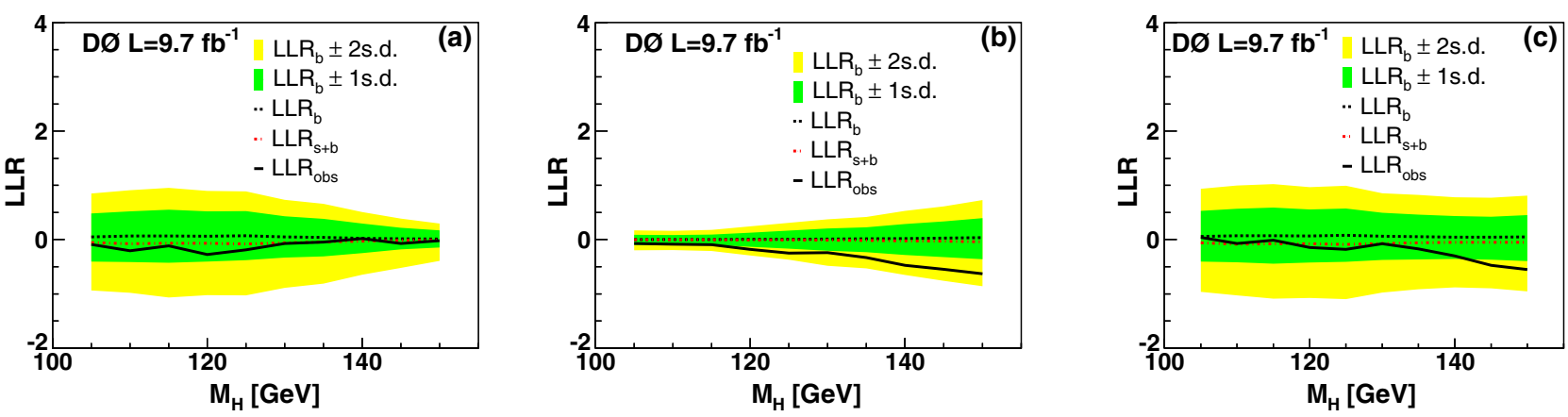

FIG. 6 (color online). LLR distributions for (a) the T subsample, (b) the W subsample, and (c) the sum of the combined T and W subsamples. The $\mu \tau j j$ and $e \tau j j$ analyses are combined. The black dashed line shows the expected LLR for the background-only hypothesis and the red dash-dotted line shows the expected LLR for the signal-plus-background hypothesis. The solid black line indicates the observed LLR. The green (yellow) shaded bands indicate the \pm 1 s.d. ( \pm 2 s.d.) uncertainties on the expected background-only LLR.

value, and the data events to which the distributions are to be compared are similarly provided with the test $M_{H}$ value. The global BDT approach removes the variation in the training at different $M_{H}$ values and provides a more uniform distribution of Higgs boson cross section limits with minimal $(\approx 10 \%)$ deterioration in limits relative to separate training at each mass point.

\section{Choice of BDT binning}

If the subsample BDT distributions have bins with a small number of background events, there can be statistical fluctuations in the calculated limits. These are reduced by choosing BDT bin sizes that ensure that all bins have at least 20 background events before application of event weights.

Representative final discriminants are shown for the $\mathrm{T}$ subsample (at $M_{H}=125 \mathrm{GeV}$ ) and the $\mathrm{W}$ sample (at $M_{H}=145 \mathrm{GeV}$ ) in Figs. 4 and 5 for the $\mu \tau j j$ and $e \tau j j$ analyses, respectively. The agreement of predicted backgrounds and the observed data is good.

\section{SYSTEMATIC UNCERTAINTIES}

A large number of systematic uncertainties have been considered, often broken down separately by analysis channel and subsample, tau type, or background or signal process. The luminosity and trigger uncertainties are obtained from separate analyses of D0 data. The lepton and tau identification uncertainties are obtained from special samples enriched in $Z$ boson decays. The jet energy scale, energy resolution, identification and vertex confirmation uncertainties are obtained from special dijet and $\gamma+$ jet samples separately for the $\mathrm{T}$ and $\mathrm{W}$ subsamples. Uncertainties in the SM background cross section normalizations and shapes are obtained using theoretical uncertainties, and the extent to which special data samples enriched in each background process agree with MC predictions. The MJ background uncertainties are determined by comparing the results using the MJ-enriched samples with those obtained using the SS signal sample after SM background subtraction.

Signal cross section uncertainties are obtained from theoretical estimates and include the effect of PDF uncertainties. Table IV summarizes the systematic uncertainties on the various sources. For each source, the impact on the final discriminant is assessed by changing the appropriate parameters by 1 s.d. from the nominal values. Some of the uncertainties affect only the normalization of the final discriminant distribution and some modify its shape while keeping the normalization fixed.
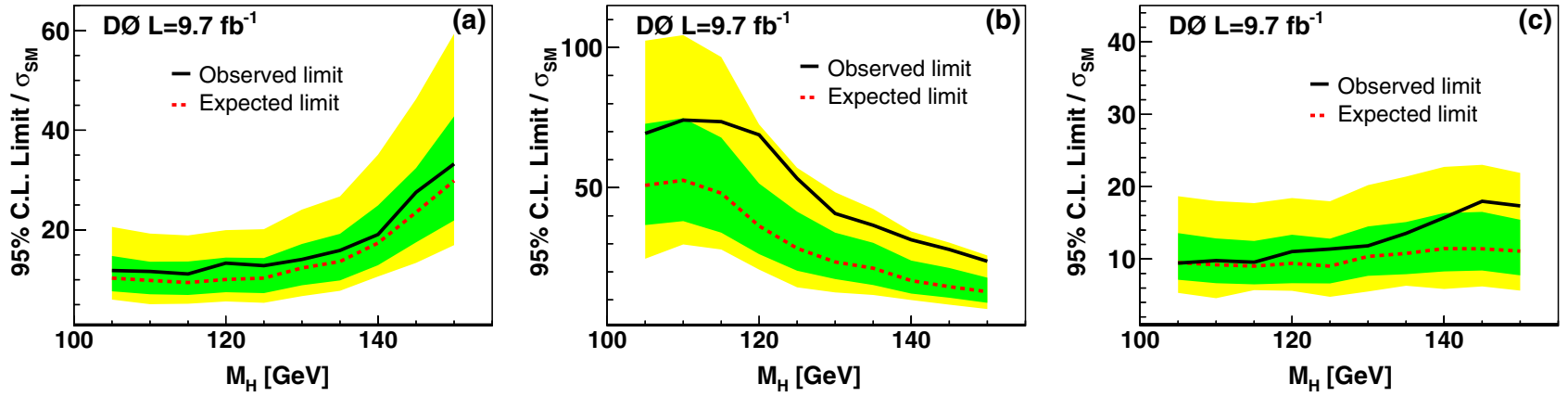

FIG. 7 (color online). The ratio of 95\% C.L. upper limits on Higgs boson production for the (a) T subsample, (b) W subsample, and (c) the sum of the $\mathrm{T}$ and $\mathrm{W}$ subsamples. The $\mu \tau j j$ and $e \tau j j$ analyses are combined. The green (yellow) shaded bands indicate the \pm 1 s.d. ( \pm 2 s.d.) uncertainties on the expected limit ratios. 
TABLE V. The ratio of 95\% C.L. upper limits on Higgs boson production to the SM prediction.

\begin{tabular}{|c|c|c|c|c|c|c|c|c|c|c|c|c|c|c|c|c|c|c|}
\hline \multirow[b]{3}{*}{$M_{H}$} & \multicolumn{6}{|c|}{$\mu \tau j j$} & \multicolumn{4}{|c|}{$e \tau j j$} & \multicolumn{4}{|c|}{$\mu \tau j j+e \tau j j$} & \multirow{2}{*}{\multicolumn{4}{|c|}{$\mathrm{W} \quad \mathrm{T}+\mathrm{W}$}} \\
\hline & \multicolumn{2}{|c|}{$\mathrm{T}$} & \multicolumn{2}{|c|}{ W } & \multicolumn{2}{|c|}{$\mathrm{T}+\mathrm{W}$} & \multicolumn{2}{|c|}{$\mathrm{T}$} & \multicolumn{2}{|c|}{ W } & \multicolumn{2}{|c|}{$\mathrm{T}+\mathrm{W}$} & \multicolumn{2}{|l|}{$\mathrm{T}$} & & & & \\
\hline & Exp & Obs & Exp & Obs & Exp & Obs & Exp & Obs & Exp & Obs & Exp & Obs & Exp & Obs & Exp & Obs & Exp & Obs \\
\hline 105 & 15.3 & 18.4 & 116 & 84.9 & 14.1 & 16.3 & 16.3 & 14.6 & 62.3 & 107 & 14.9 & 11.8 & 10.3 & 11.8 & 50.9 & 69.3 & 9.4 & 9.4 \\
\hline 110 & 14.3 & 18.2 & 113 & 112 & 13.9 & 16.8 & 15.6 & 14.6 & 64.3 & 101 & 14.4 & 12.5 & 9.9 & 11.7 & 52.6 & 74.1 & 9.1 & 9.8 \\
\hline 115 & 14.6 & 17.3 & 86.6 & 121 & 13.8 & 17.2 & 14.9 & 14.8 & 66.5 & 93.3 & 14.2 & 11.7 & 9.5 & 11.2 & 48.0 & 73.5 & 9.0 & 9.5 \\
\hline 120 & 15.5 & 16.5 & 60.1 & 125 & 14.6 & 17.6 & 15.7 & 19.7 & 56.6 & 75.4 & 14.5 & 15.3 & 10.1 & 13.3 & 36.4 & 68.9 & 9.4 & 11.1 \\
\hline 125 & 15.3 & 17.0 & 48.9 & 84.9 & 14.4 & 18.4 & 15.9 & 18.7 & 46.1 & 65.0 & 14.6 & 16.0 & 10.4 & 12.8 & 28.4 & 53.3 & 9.0 & 11.3 \\
\hline 130 & 17.1 & 18.3 & 32.8 & 55.4 & 15.3 & 17.4 & 20.1 & 21.8 & 51.5 & 65.3 & 18.3 & 19.5 & 12.4 & 14.1 & 23.5 & 40.8 & 10.2 & 11.8 \\
\hline 135 & 19.1 & 19.9 & 28.8 & 53.7 & 15.6 & 21.0 & 23.6 & 25.6 & 51.0 & 51.8 & 20.4 & 21.4 & 13.7 & 15.9 & 21.3 & 36.6 & 10.8 & 13.5 \\
\hline 140 & 24.3 & 23.7 & 22.5 & 44.5 & 15.9 & 22.8 & 31.0 & 33.9 & 40.0 & 40.8 & 24.4 & 26.3 & 17.4 & 19.1 & 16.9 & 31.4 & 11.5 & 16.0 \\
\hline 145 & 30.7 & 33.9 & 19.1 & 37.3 & 15.1 & 23.7 & 42.5 & 47.6 & 45.5 & 44.0 & 29.8 & 36.0 & 23.5 & 27.6 & 14.7 & 27.9 & 11.5 & 17.9 \\
\hline 150 & 38.1 & 36.0 & 17.5 & 34.8 & 14.9 & 22.2 & 58.4 & 69.5 & 29.0 & 27.5 & 24.8 & 30.8 & 29.9 & 33.3 & 12.9 & 23.7 & 11.1 & 17.2 \\
\hline
\end{tabular}

\section{CROSS SECTION LIMITS}

The upper limits on the Higgs boson cross section for each analysis are obtained from the final discriminants for Higgs boson masses between 105 and $150 \mathrm{GeV}$ in $5 \mathrm{GeV}$ increments obtained with the modified frequentist method of Ref. [38], using a negative log likelihood ratio (LLR) for the background-only and signal-plus-background hypotheses as the test statistic. The LLR plots, individually for T and $\mathrm{W}$ subsamples and for their sum, are shown in Fig. 6.

The impact of systematic uncertainties on the limits is reduced by maximizing a "profile" likelihood function [39] in which these uncertainties are constrained to Gaussian priors to give a best fit to the data. The appropriate correlations are retained (for example, the $\mathrm{VH}$ cross section uncertainty is fully correlated across the $\mu \tau j j$ and $e \tau j j$ analyses and the $\mathrm{T}$ and $\mathrm{W}$ subsamples). The value of the Higgs boson cross section is adjusted in each limit calculation until the value of $C L_{s}$ reaches 0.05 , corresponding to the $95 \%$ C.L., where $C L_{s}=C L_{s+b} / C L_{b}$ and $C L_{s+b}$ $\left(C L_{b}\right)$ are the probabilities for the negative LLR value observed in simulated signal + background (background) pseudoexperiments to be less signal-like than that observed in our data. The ratio of the resulting 95\% C.L. upper limits to the SM predictions on the production times branching ratio are shown in Fig. 7 and in Table V.
In summary we have searched for the SM Higgs boson in final states involving an electron or a muon and a hadronically decaying tau, together with at least two jets. We set $95 \%$ C.L. limits on the ratio of the Higgs boson production cross section to that predicted in the SM of 11.3 times for a Higgs boson mass of $125 \mathrm{GeV}$, to be compared with an expected ratio of 9.0. For a subsample enriched in $H \rightarrow \tau \tau$ decays we observe (expect) a ratio of 12.8 (10.4) for $M_{H}=125 \mathrm{GeV}$. These are the most stringent limits on Higgs boson production with $H \rightarrow \tau \tau$ decay at the Tevatron to date. For an orthogonal subsample enriched in $H \rightarrow W W$ decays the corresponding ratios are 14.7 (11.5) at $M_{H}=145 \mathrm{GeV}$.

\section{ACKNOWLEDGMENTS}

We thank the staffs at Fermilab and collaborating institutions, and acknowledge support from the DOE and NSF (USA); CEA and CNRS/IN2P3 (France); MON, Rosatom and RFBR (Russia); CNPq, FAPERJ, FAPESP and FUNDUNESP (Brazil); DAE and DST (India); Colciencias (Colombia); CONACyT (Mexico); NRF (Korea); FOM (The Netherlands); STFC and the Royal Society (United Kingdom); MSMT and GACR (Czech Republic); BMBF and DFG (Germany); SFI (Ireland); The Swedish Research Council (Sweden); and CAS and CNSF (China).
[1] R. Barate et al. (LEP Working Group for Higgs boson searches), Phys. Lett. B 565, 61 (2003).

[2] T. Aaltonen et al. (CDF and D0 Collaborations), Phys. Rev. Lett. 104, 061802 (2010); this issue, Phys. Rev. D 88, 052014 (2013).

[3] G. Aad et al. (ATLAS Collaboration), Phys. Lett. B 716, 1 (2012).
[4] S. Chatrchyan et al. (CMS Collaboration), Phys. Lett. B 716, 30 (2012).

[5] M. Baak, M. Goebel, J. Haller, A. Hoecker, D. Kennedy, K. Mönig, M. Schott, and J. Stelzer (GFitter collaboration), Eur. Phys. J. C 72, 2003 (2012).

[6] V. M. Abazov et al. (D0 Collaboration), Phys. Rev. Lett. 109, 121802 (2012). 
[7] T. Aaltonen et al. (CDF Collaboration), Phys. Rev. Lett. 109, 111802 (2012).

[8] T. Aaltonen et al. (CDF and D0 Collaborations), Phys. Rev. Lett. 109, 071804 (2012).

[9] V. M. Abazov et al. (D0 Collaboration), Phys. Lett. B 714, 237 (2012)

[10] T. Aaltonen et al. (CDF Collaboration), Phys. Rev. Lett. 108, 181804 (2012).

[11] S. Abachi et al. (D0 Collaboration), Nucl. Instrum. Methods Phys. Res., Sect. A 338, 185 (1994).

[12] V. M. Abazov et al. (D0 Collaboration), Nucl. Instrum. Methods Phys. Res., Sect. A 565, 463 (2006).

[13] V. M. Abazov et al., Nucl. Instrum. Methods Phys. Res., Sect. A 552, 372 (2005).

[14] S. M. Ahmed et al., Nucl. Instrum. Methods Phys. Res., Sect. A 634, 8 (2011).

[15] R. Angstadt et al., Nucl. Instrum. Methods Phys. Res., Sect. A 622, 298 (2010).

[16] B. C. K. Casey et al., Nucl. Instrum. Methods Phys. Res., Sect. A 698, 208 (2013).

[17] J. Pumplin, D. R. Stump, J. Huston, H-L. Lai, P. Nadolsky, and W-K. Tung, J. High Energy Phys. 07 (2002) 012.

[18] M. L. Mangano, F. Piccinini, A. D. Polosa, M. Moretti, and R. Pittau, J. High Energy Phys. 07 (2003) 001; we use ALPGEN version 2.05.

[19] T. Sjöstrand et al., arXiv:hep-ph/0308153.

[20] V. M. Abazov et al. (D0 Collaboration), Phys. Rev. Lett. 100, 102002 (2008).

[21] K. Melnikov and F. Petriello, Phys. Rev. D 74, 114017 (2006).

[22] R. Hamburg, W. L. van Neerven, and T. Matsuura, Nucl. Phys. B359, 343 (1991); B644, 403 (2002).

[23] A. D. Martin, W. J. Stirling, R. S. Thorne, and G. Watt, Eur. Phys. J. C 63, 189 (2009).

[24] E. Boos, V.E. Bunichev, L. V. Dudko, V.I. Savrin, and V. V. Sherstnev, Phys. At. Nucl. 69, 1317 (2006); E. Boos, V. Bunichev, M. Dubinin, L. Dudko, V. Edneral, V. Ilyin, A. Kryukov, V. Savrin, A. Semenov, and A. Sherstnev
(CompHEP Collaboration), Nucl. Instrum. Methods Phys. Res., Sect. A 534, 250 (2004).

[25] N. Kidonakis, Phys. Rev. D 74, 114012 (2006); U. Langenfeld, S. Moch, and P. Uwer, Phys. Rev. D 80, 054009 (2009).

[26] J. M. Campbell, R. K. Ellis, and C. Williams, MCFMMonte Carlo for FeMtobarn processes, http:// mcfm.fnal.gov; J. M. Campbell, arXiv:hep-ph/0105226.

[27] A. Djouadi, J. Kalinowski, and M. Spira, Comput. Phys. Commun. 108, 56 (1998).

[28] S. Jadach, Z. Was, R. Decker, and J. H. Kühn, Comput. Phys. Commun. 76, 361 (1993).

[29] R. Brun and F. Carminati, CERN Program Library Long Writeup W5013, 1993 (unpublished).

[30] V. M. Abazov et al. (D0 Collaboration), Phys. Lett. B 670, 292 (2009).

[31] G. Blazey et al., in Proceedings of the Workshop: QCD and Weak Boson Physics in Run II, edited by U. Baur, R. K. Ellis, and D. Zeppenfeld (Report No. Fermilab-Pub00/297, 2000).

[32] A. Schwartzman, Report No. FERMILAB-THESIS-200421 (2004).

[33] A. Hoecker et al., Proc. Sci., ACAT (2007) 040.

[34] L. Breiman, J. Friedman, C. J. Stone, and R. A. Olshen, Classification and Regression Trees (Wadsworth, Stamford, 1984).

[35] D. Bowser-Chao and D. L. Dzialo, Phys. Rev. D 47, 1900 (1993).

[36] C. Gini, Variabilità e Mutabilità (1912); reprinted in Memorie di Metodologica Statistica, edited by E. Pizetti and T. Salvemini (Libreria Eredi Virgilio Veschi, Rome, 1955).

[37] A. Elain, P. Murat, A. Pranko, and A. Safanov, Nucl. Instrum. Methods Phys. Res., Sect. A 654, 481 (2011).

[38] A. Read, J. Phys. G 28, 2693 (2002); T. Junk, Nucl. Instrum. Methods Phys. Res., Sect. A 434, 435 (1999).

[39] W. Fisher, Report No. FERMILAB-TM-2386-E (2007). 